\title{
HIV-antibody complexes enhance production of type I interferon by plasmacytoid dendritic cells
}

\author{
Rebecca T. Veenhuis, ${ }^{1}$ Zachary T. Freeman, ${ }^{1}$ Jack Korleski, ${ }^{1}$ Laura K. Cohen, ${ }^{1}$ Guido Massaccesi, ${ }^{1}$ Alessandra Tomasi, ${ }^{1}$ \\ Austin W. Boesch, ${ }^{2}$ Margaret E. Ackerman, ${ }^{2}$ Joseph B. Margolick, ${ }^{3}$ Joel N. Blankson, ${ }^{1}$ Michael A. Chattergoon, ${ }^{1}$ and Andrea L. Cox ${ }^{1}$ \\ 'Department of Medicine, Johns Hopkins University School of Medicine, Baltimore, Maryland, USA. ${ }^{2}$ Thayer School of Engineering, Dartmouth College, Hanover, New Hampshire, USA. ${ }^{3}$ Johns Hopkins \\ Bloomberg School of Public Health, Baltimore, Maryland, USA.
}

\begin{abstract}
Type I IFN production is essential for innate control of acute viral infection; however, prolonged high-level IFN production is associated with chronic immune activation in HIV-infected individuals. Although plasmacytoid DCs (pDCs) are a primary source of IFN, the mechanisms that regulate IFN levels following the acute phase are unknown. We hypothesized that HIV-specific Ab responses regulate late IFN production. We evaluated the mechanism through which HIV-activated pDCs produce IFN as well as how both monoclonal HIV-specific Abs and Abs produced in natural HIV infection modulated normal pDC sensing of HIV. We found that HIV-induced IFN production required TLR7 signaling, receptor-mediated entry, fusion, and viral uncoating, but not endocytosis or HIV life cycle stages after uncoating. Abs directed against the HIV envelope that do not interfere with CD4 binding markedly enhanced the IFN response, irrespective of their ability to neutralize CD4+ T cell infection. Ab-mediated enhancement of IFN production required Fc $\gamma$ receptor engagement, bypassed fusion, and initiated signaling through both TLR7 and TLR9, which was not utilized in the absence of Ab. Polyclonal Abs isolated from HIV-infected subjects also enhanced pDC production of IFN in response to HIV. Our data provide an explanation for high levels of IFN production and immune activation in chronic HIV infection.
\end{abstract}

\section{Introduction}

Type I IFN production is essential in innate control of acute viral infection, but can enhance pathogenesis during chronic infections such as HIV, SIV, and lymphocytic choriomeningitis virus infection (1-3). In HIV and SIV infection, persistent high-level IFN production is associated with chronic immune activation, including higher levels of activation molecule expression, increased immune activation gene expression, and/or more rapid CD4 depletion (4, 5). Increased circulating IFN during the chronic stage of HIV infection has been associated with rapid progression to $\operatorname{AIDS}(6,7)$.

Both sustained and high-level expression of IFN-stimulated genes (ISGs) have been linked to the development of chronic immune activation and AIDS in SIV-infected monkeys, while a rapid return to baseline ISG production and lower ISG expression are both associated with asymptomatic infection $(8,9)$. While blockade of IFN responses prior to SIV infection led to rapid disease progression and early exogenous IFN administration prevented systemic infection, prolonged administration induced IFN desensitization (4). Additionally, the blockade of IFN signaling during chronic infection has been shown to restore immune function, decrease the size of the HIV reservoir, and delay viral rebound after antiretroviral therapy (ART) interruption $(10,11)$. Thus, the role of IFN in HIV/SIV infection is dichotomous, with

Authorship note: M.A. Chattergoon and A.L. Cox contributed equally to the work. Conflict of interest: The authors have declared that no conflict of interest exists. Submitted: June 1, 2017; Accepted: September 21, 2017.

Reference information: J Clin Invest. 2017;127(12):4352-4364.

https://doi.org/10.1172/JCI95375. robust IFN responses controlling infection in the acute phase and sustained high-level IFN production associated with enhanced pathogenesis in the chronic phase.

Nonneutralizing Abs also seem to play a dichotomous role in the control of chronic infections, depending on disease stage. For example, nonneutralizing Abs have distinctive profiles in latent versus active tuberculosis (TB). Abs isolated from people with latent TB infection (LTBI) are associated with unique functional profiles, selective binding to $\mathrm{Fc} \gamma$ receptor 3 ( $\mathrm{Fc} \gamma \mathrm{R} 3$ ), and distinct glycosylation patterns. When compared with Abs isolated from people with active $\mathrm{TB}$, nonneutralizing Abs from people with LTBI were shown to better enhance phagolysosomal maturation, inflammasome activation, and macrophage killing of intracellular Mycobacterium tuberculosis, suggesting an important role for Abs in controlling TB pathogenesis (12). Nonneutralizing Abs have important roles in chronic viral infections as well. They are necessary to clear early high-dose LCMV infection in mice in the absence of IL-10 signaling and provide protection upon adoptive transfer prior to infection (13). Results from the RV144 prophylactic HIV vaccine trial and Ad26 vaccination in nonhuman primates argue that some degree of protection from HIV/SIV infection can be achieved in the absence of neutralizing Abs and $\mathrm{T}$ cell responses $(14,15)$. These data suggest that the functional role of Abs outside of neutralization in HIV should be further investigated.

Despite its importance in pathogenesis, the mechanisms that regulate the level of IFN production after the acute phase of HIV remain unknown as does the role in IFN regulation of HIV-specific Abs that do not block CD4 binding. Plasmacytoid DCs (pDCs) are the principal producers of IFN, specifically type I 
A

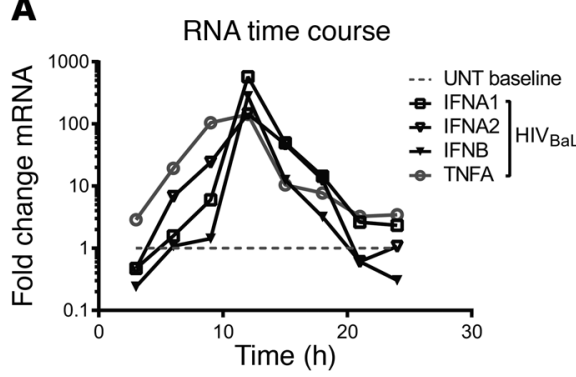

B

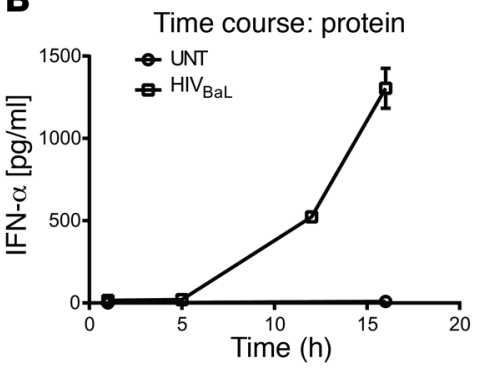

C

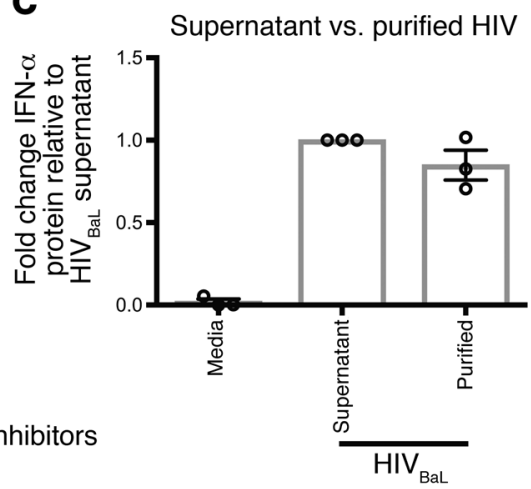

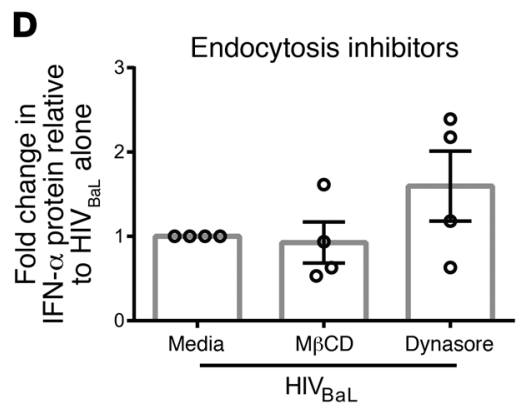

E

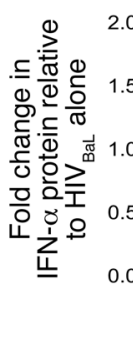

HIV life cycle inhibitors

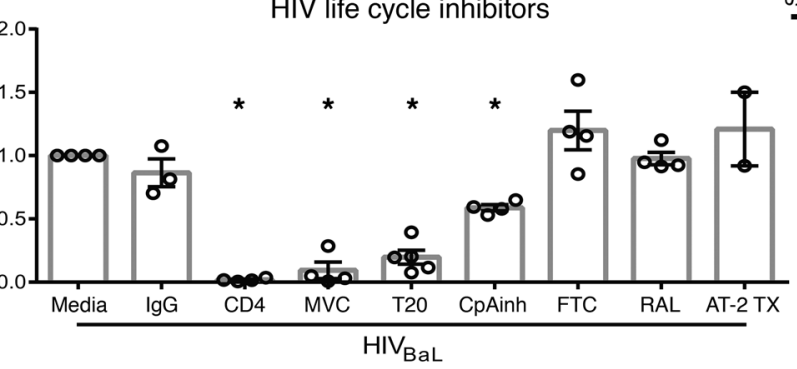

Figure 1. Type I IFN induction by HIV requires receptor-mediated entry, not endocytosis or productive infection. After culture with HIV ${ }_{\text {BaL' }}$ (A) human pDCs were harvested and assessed for type I IFN and TNF- $\alpha$ mRNA expression and (B) IFN- $\alpha$ protein production was measured at multiple time points. UNT, untreated with HIV. (A and B) One representative experiment, completed in triplicate, is shown. (C) Human pDCs were cultured with either HIV ${ }_{\text {BaL }}$ culture supernatant or with purified $\mathrm{HIV}_{\text {BaL }}$ for 15 hours, then assessed for IFN- $\alpha$ protein production $(n=3)$. (D) Human pDCs were cultured with endocytosis

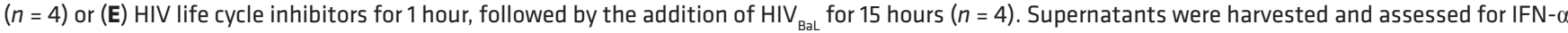
protein production. Each data point indicates the average IFN- $\alpha$ production from 1 donor's pDCs tested in at least duplicate and normalized to the mediaalone condition. Error bars and gray boxes represent the SEM and the mean, respectively. Conditions were compared using 1-way ANOVA with Dunnett's multiple comparisons test. ${ }^{*} P<0.01$.

IFN- $\alpha / \beta$. HIV is sensed by pDCs through endosomal TLR7 recognition of RNA $(16,17)$. In this study, we further define the mechanism through which HIV activates pDCs to produce IFN and demonstrate that both mAbs directed against sites outside the CD4-binding sites and Abs generated in people with persistent HIV infection enhance IFN production by pDCs. This substantial enhancement of pDC IFN production by Abs produced in natural HIV infection may contribute to the persistent IFN elevation and immune activation observed after the humoral response develops in chronic HIV infection.

\section{Results}

pDCs require receptor-mediated entry and uncoating of HIV to produce IFN. Purified human pDCs produced IFN (Figure 1, A and B) when incubated with culture supernatants from the HIV culture strain $\mathrm{HIV}_{\mathrm{BaL}}$. Adding exogenous IFN- $\alpha$ or IFN- $\beta$ to $\mathrm{pDCs}$ did not induce pDCs to produce IFN (Supplemental Figure 1; supplemental material available online with this article; https://doi.org/10.1172/ JCI95375DS1). Conversely, coculturing pDCs with purified $\mathrm{HIV}_{\mathrm{BaL}}$ produced the same amount of IFN as coculturing pDCs with culture supernatant, indicating that the virus rather than other factors in culture supernatant induced pDC IFN production (Figure 1C). To test whether endocytosis of virus was required for $\mathrm{pDC}$ activation, as previously demonstrated for other innate immune responses to HIV (18), we repeated the assay in the presence of 2 endocytosis inhibitors, M $\beta C D$ (an inhibitor of clathrin-mediated endocytosis) and Dynasore (a dynamin inhibitor that results in inhibition of both clathrin and caveolar-mediated endocytosis).
We saw no effect of either inhibitor, demonstrating that endocytosis was not required to activate IFN production (Figure 1D). This led us to hypothesize that HIV enters pDCs though binding specific viral receptors and fusion. To test this, we used inhibitors of HIV receptor (CD4) binding, coreceptor (CCR5) binding, or fusion. We observed that blockade of the HIV-CD4 interaction with anti-CD4 $\mathrm{mAb}$, of CCR5 coreceptor binding with the CCR5 antagonist maraviroc (MVC), or of fusion with enfuvirtide (T20) all significantly diminished $\mathrm{HIV}_{\mathrm{BaL}}$-driven IFN production by pDCs versus $\mathrm{HIV}_{\mathrm{BaL}}$ alone (Figure 1E). These results support that HIV must bind CD4 and CCR5 and fuse with the pDC membrane to activate pDC IFN production. We then assessed what steps are required after HIV binding and cell fusion. Cyclophilin A mediates uncoating of the HIV viral capsid core (19). Partial inhibition with a cyclophilin A inhibitor (CypA) resulted in a significant $(P<0.01)$ reduction of IFN production (Figure 1E). These data in total suggest that HIV enters pDCs via receptor/coreceptor binding and fusion followed by uncoating, rather than via endocytosis.

To determine whether later stages of the HIV life cycle were necessary for IFN production, we cultured human pDCs with $\mathrm{HIV}_{\mathrm{BaL}}$ in the presence of reverse-transcriptase (emtricitabine [FTC]) and integrase (Raltegravir [RAL]) inhibitors or with $\mathrm{HIV}_{\mathrm{BaL}}$ deactivated with 2, 2-dithiodipyridine aldrithiol-2 (AT-2). AT-2 treatment maintains surface viral protein conformation and function, but arrests HIV replication before initiation of reverse transcription (20). None of these treatments reduced IFN production by pDCs, demonstrating that HIV life cycle stages following uncoating are not required for the IFN response (Figure 1E). 

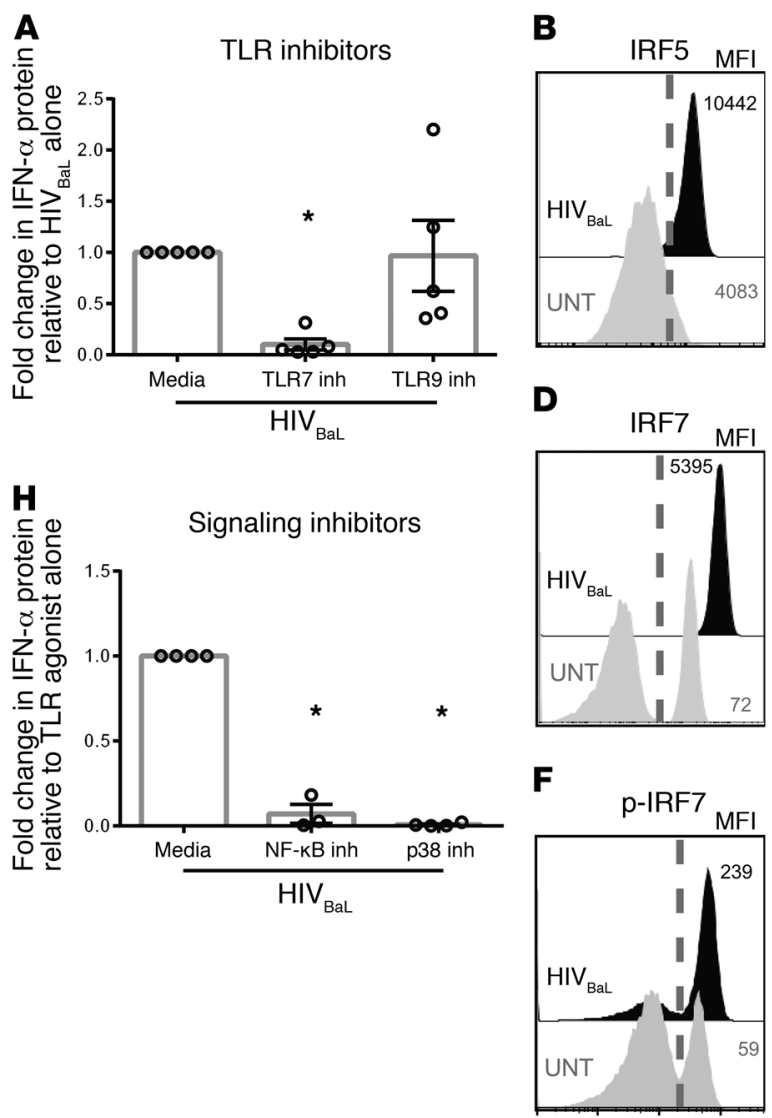
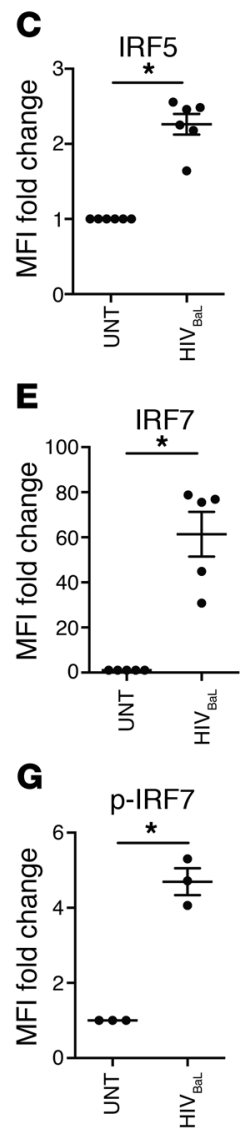

Figure 2. pDCs utilize TLR7, IRF7, IRF5, NF-KB, and MAPK signaling to produce IFN in response to HIV. (A) Human pDCs were cultured with TLR inhibitors for 1 hour, followed by the addition of HIV BaL for 15 hours. Supernatants were harvested and assessed for IFN- $\alpha$ protein production $(n=5)$. Human pDCs were cultured with $\mathrm{HIV}_{\text {BaL }}$ (black histogram) or without HIV $_{\text {BaL }}$ (gray histogram) for 15 hours per cell and were then permeabilized and stained for (B and C) IRF5, (D and E) IRF7, or (F and $\mathbf{G}$ ) $\mathrm{p}$-IRF7. The dashed gray line indicates the gate cut-off for the positive IRF5, IRF7, or p-IRF7 populations. (B, D, and F) Representative histograms are shown. (C, E, and $\mathbf{G})$ Summary graphs of at least 3 independent experiments are shown. (H) Human pDCs were cultured with signaling inhibitors for 1 hour, followed by the addition of HIV for 15 hours. Supernatants were harvested and assessed for IFN- $\alpha$ protein production $(n=3)$. Each data point indicates the average IFN- $\alpha$ production from 1 donor's pDCs tested in at least duplicate and normalized to the media condition. Error bars and gray boxes represent the SEM and the mean, respectively. Conditions were compared using 1-way ANOVA with Dunnett's multiple comparisons test (A and $\mathbf{H})$ or Student's $t$ test $(\mathbf{C}, \mathbf{E}, \mathbf{G}) .{ }^{*} P<0.01$.
Receptor/coreceptor blockade and HIV life cycle inhibitors had no effect on PDC production of IFN in response to the TLR7 agonist resiquimod, confirming the specific effect of receptor/ coreceptor blockade on HIV sensing and the absence of global impairment of pDC function (Supplemental Figure 2). In sum, these data show that receptor-mediated entry and uncoating are required for pDCs to release IFN in response to HIV, but not endocytosis or HIV life cycle stages after uncoating.

$H I V$ signals via TLR7, IRF7, and IRF5 and requires the $N F-\kappa B$ and MAPK pathways to induce IFN. Next, we sought to determine how HIV triggers TLR signaling following uncoating. To determine TLR usage, we cultured pDCs and $\mathrm{HIV}_{\mathrm{BaL}}$ in the presence of antagonists of TLR7 and TLR9, the only TLRs present in human pDCs (21). Specificity of the TLR antagonists was confirmed by blocking of the actions of the TLR7 agonist resiquimod and the TLR9 agonist ODN2216 (Supplemental Figure 3). We observed a significant decrease in HIV induction of IFN in the presence of the TLR7 antagonist, but not the TLR9 antagonist, demonstrating dependence on TLR7 (Figure 2A). Because the adaptor protein IRF7 is essential and IRF5 is also utilized in downstream TLR7 signaling, we then assessed bulk IRF5 and IRF7 levels and phosphorylation of IRF7 after HIV-induced pDC activation $(22,23)$. There was a significant increase in total IRF5, total IRF7, and phosphorylated IRF7 (p-IRF7) with HIV activation (Figure 2, B-G and Supplemental Figure 5) compared with pDCs not cultured with HIV. Phosphorylated protein gating was determined by comparing the untreated condition with the HIV-stimulated condition (Supplemental Figure 4). The downstream signaling pathways for IRF5 and IRF7 overlap, and there are no flow cytometry reagents available to assess p-IRF5. Therefore, we assessed cells with p-IRF7 for further analysis of the signaling required downstream of IRF5/7. Cells with increased p-IRF7 also had increased levels of phosphorylated p65 and of the JNK and p38 MAPK family members (Supplemental Figure 5A). STAT1 was used as a positive control because IFN receptor engagement induces STAT1 phosphorylation $(24,25)$. TBK1 was used as a negative control because TBK1 is in the IRF3, not the IRF7, signaling pathway (26). As predicted and confirming the irrelevance of IRF3, HIV-activated pDCs had high levels of phosphorylated STAT1 with no phosphorylated TBK1 (Supplemental Figure 5B). To assess whether the increases in $\mathrm{p} 65$ and p38 MAPK phosphorylation were directly involved in HIVinduced IFN production, we used an inhibitor of Iк-B kinase $\beta$ (IKK $\beta$ ) and a 38 MAPK inhibitor to block NF- $\mathrm{B}$ and MAPK signaling, respectively. We saw that inhibition of either NF- $\mathrm{KB}$ or MAPK signaling completely suppressed IFN production, prevented phosphorylation of p65 and p38 MAPK, and decreased p-IRF7 levels to baseline (Figure $2 \mathrm{H}$ and Supplemental Figure 5, C and D). As assessed by flow cytometry, the pDCs were still alive and functional in the presence of these inhibitors, producing IFN in response to the TLR7 agonist resiquimod (Supplemental Figure 6). Interestingly, inhibition of MAPK signaling did not prevent IFN production in response to resiquimod, but inhibition of NF- $\mathrm{KB}$ signaling completely suppressed IFN production (Supplemental Figure 6). This suggests that MAPK signaling is critical for HIVinduced, but not all, IFN production by pDCs. In sum, these data demonstrate that HIV activates the IFN response by first engaging 
A

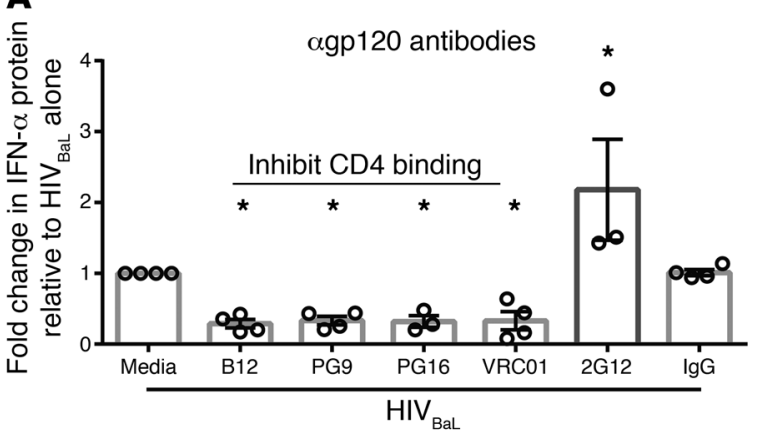

B

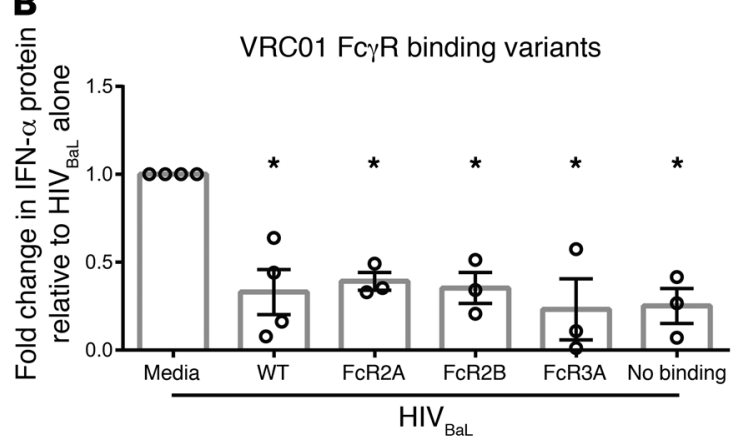

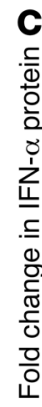
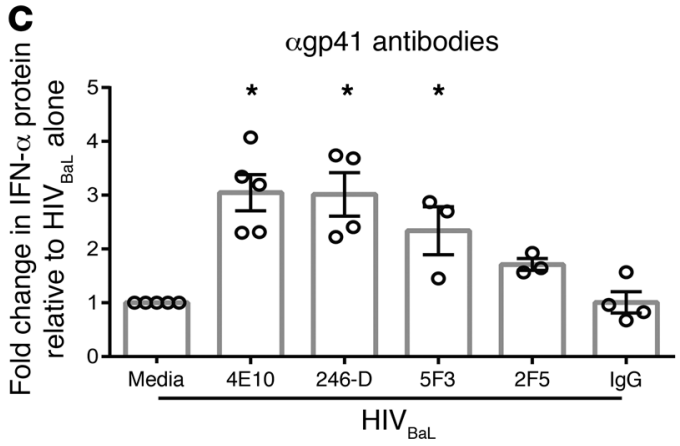

D

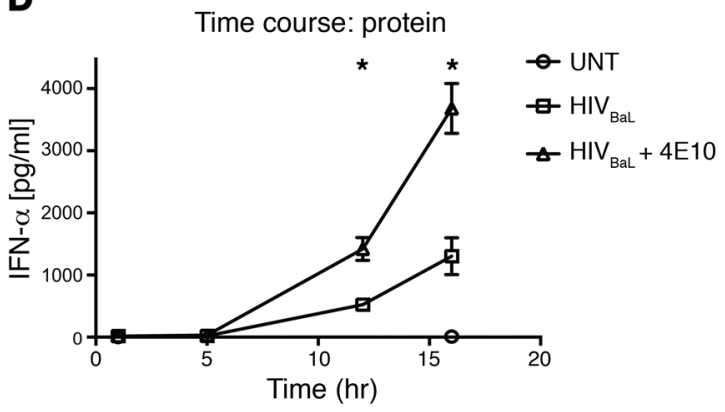

Figure 3. HIV Ab specificity regulates type I IFN production by pDCs. HIV ${ }_{B a L}$ was cultured with (A) anti-gp120 Abs that interfere with CD4 binding (B12, PC9, PG16, and VRC01) or with an anti-gp120 Ab that does not interfere with CD4-binding (2G12), (B) VRC01 Fc $\gamma R$-binding variants that either enhanced Fc binding to the designated Fc $\gamma R$ or prevented all Fc $\gamma R$ binding, or (C) anti-gp41 Abs for 1 to 2 hours, which were then added to pDCs. Supernatants were harvested

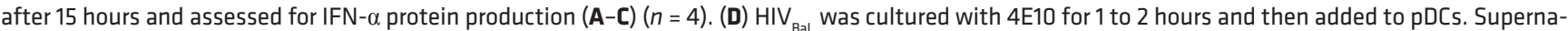
tants were harvested and assessed for IFN- $\alpha$ protein production at 1, 5, 12, and 16 hours. Data from 1 representative experiment completed in triplicate. Each data point indicates the average IFN- $\alpha$ production from 1 donor's $\mathrm{pDCs}$ tested in at least duplicate and normalized to media conditions. Error bars and gray boxes represent SEM and the mean, respectively. Conditions were compared using 1-way ANOVA with Dunnett's multiple comparisons test. ${ }^{*} P<0.05$.

TLR7, which then signals through NF-אB, MAPK, and IRF7, resulting in IFN production.

HIV-specific Abs modulate IFN production by pDCs. Because high-level IFN production is associated with pathogenesis in the chronic phase of HIV, we hypothesized that HIV-specific Abs, which develop after the most acute phase of infection, may modulate IFN production. Our data demonstrated that pDC activation by HIV depends on binding CD4, suggesting that Abs that block the CD4-HIV interaction would block HIV sensing. As predicted, all of the tested mAbs known to interfere with CD4 binding to the HIV envelope protein gp120 (B12, PG9, PG16, and VRC01) did significantly reduce IFN production by pDCs (Figure 3A and Supplemental Table 1) compared with HIV with media alone. To verify that it is the capacity to disrupt CD4 binding that mediates suppression, we tested an additional neutralizing $\mathrm{mAb}$ that binds to gp120, but does not disrupt CD4 binding: 2G12. The $2 \mathrm{G} 12 \mathrm{mAb}$ did not suppress IFN production by pDCs, confirming that disruption of CD4 binding to gp120 was required for Abmediated suppression of IFN production (Figure 3A). We hypothesized that because it is the Fab portions' capacity to block CD4 binding that determines suppression, there was no dependence on Fc $\gamma \mathrm{R}$ engagement. To assess this, we compared suppression with WT VRCO1 to that of variants of the VRC01 mAb. These variants have identical antigen binding (Fab) portions, but Fc region mutations that resulted either in preferential binding to distinct Fc $\gamma$ Rs or in no binding to any Fc $\gamma R$, depending on the mutation (27). Modification of the Fc region of VRC01 to alter or negate Fc $\gamma$ R engagement did not impair the capacity of VRC01 to suppress IFN production, demonstrating that Fc $\gamma \mathrm{R}$ engagement is not required for Ab-mediated suppression of IFN production by pDCs (Figure 3B). In sum, mAbs that bind HIV at the CD4-binding site of gp120 prevent HIV stimulation of pDC IFN via a Fab-dependent, FcR-independent process, consistent with simple blockade of HIV engagement of its CD4 receptor.

To further assess the effect of mAbs that bind the HIV envelope but don't disrupt CD4 binding, we compared IFN production induced by $\mathrm{HIV}_{\mathrm{BaL}}$ alone to that induced by $\mathrm{HIV}_{\mathrm{BaL}}$ in the presence of mAbs specific for the gp41 portion of the HIV envelope (4E10, 246-D, 5F3, and 2F5). Interestingly, we saw that all of the gp41-specific mAbs tested enhanced IFN production (Figure 3C). Two of the anti-gp41 mAbs tested (246-D and 5F3) did not neutralize HIV infection in CD4 T cells, demonstrating that enhancement of pDC production of IFN was independent of the ability to neutralize infection $(28,29)$. Additionally, the HIV region targeted by gp41-specific mAbs does not determine the level of enhancement, since the mAbs $4 \mathrm{E} 10$ and 2F5 bind very similar epitopes on the membrane proximal external region (MPER) of gp41, but have very different capacities to enhance (Figure 3C and Supplemental Table 1). To assess whether HIV-mAb complexes resulted in the earlier production of IFN protein and therefore increased total IFN production, we assessed IFN protein levels at 1, 5, 12, and 16 hours after incubation. We observed that IFN was produced at 

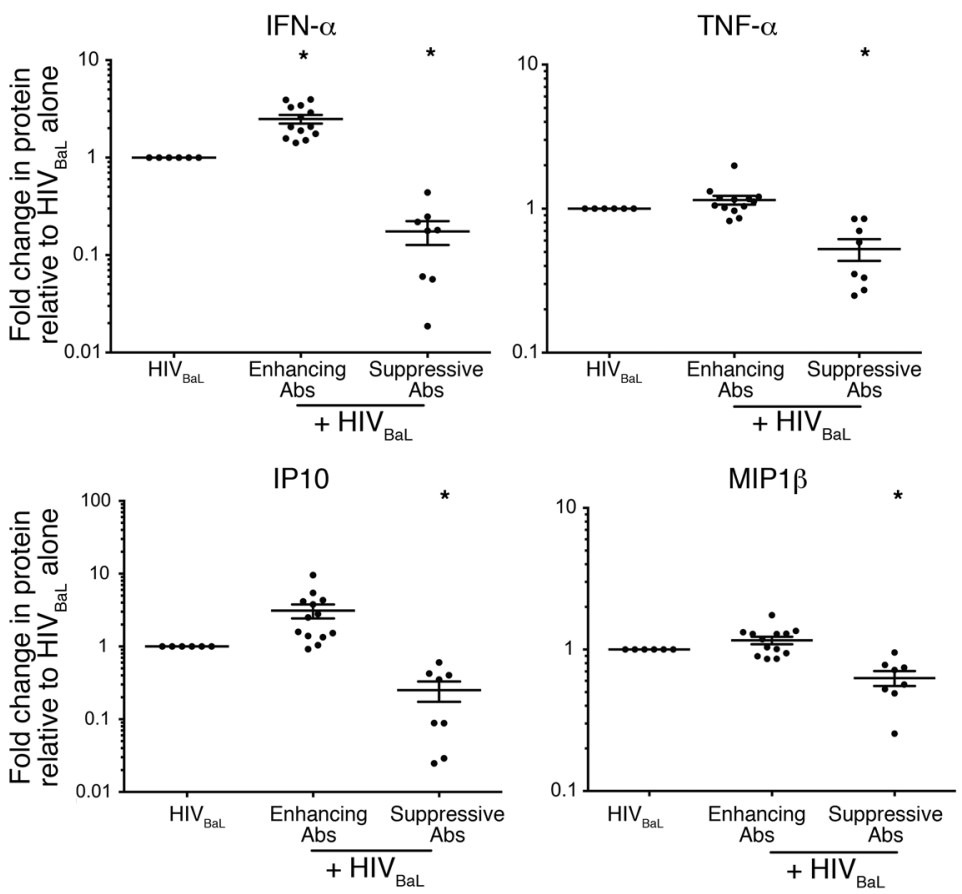

Figure 4. HIV-immune complexes that do not interfere with CD4 binding specifically enhance IFN- $\alpha$ production by $\mathrm{PDCs}$ while suppressive mAbs block production of multiple cytokines. HIV was cultured with a single enhancing Ab (4E10, 2F5, 246-D, 5F3), a single suppressive $A b$ (B12, PG9, PG16, VRC01), or no $A b$ for 1 to 2 hours and then added to $\mathrm{PDCs}$. Supernatants were harvested after 15 hours and assessed for IFN- $\alpha$, TNF- $\alpha$, IP10, and MIP1 $\beta$ protein production. Abs were grouped for analysis based on their ability to enhance or suppress independently. Each data point indicates the protein production from 1 replicate normalized to the media condition. Supernatants were tested from 3 independent donors, with 2 replicates each. Error bars represent the SEM and the mean. Conditions were compared using 1-way ANOVA with Dunnett's multiple comparisons test. ${ }^{*} P<0.05$. the same time (between 5 and 10 hours after incubation) with and without mAbs, but at higher levels at all time points with HIV-mAb complexes present versus HIV alone (Figure 3D).

We observed a titratable effect on pDC IFN mRNA and protein production by both the enhancing gp $41 \mathrm{mAbs}$ and suppressive gp120 mAbs (Supplemental Figures 7 and 8, respectively). To test whether the presence of HIV-specific mAbs had an effect on pDC production of IFN in the absence of HIV, we cocultured the cells with mAbs alone and saw no IFN induction in pDCs by mAbs in the absence of HIV (Supplemental Figure 9).

Finally, to assess whether mAbs against HIV specifically modulate the IFN response to HIV or cause global changes in pDC cytokine production, we tested supernatants from pDC cultures for 16 additional analytes (Figure 4 and Supplemental Figure 10). Of all 17 analytes tested, IFN was the only cytokine significantly enhanced by HIV-specific Abs that did not interfere with CD4 binding (Figure 4, Enhancing Abs). However, 3 analytes, TNF- $\alpha$, IP10, and MIP-1 $\beta$, were suppressed by HIV-specific Abs that block CD4 binding (Figure 4, Suppressive Abs), providing further evidence that Abs that interfere with CD4 engagement prevent the virus from engaging $\mathrm{pDCs}$ and inducing an antiviral response. Of the 13 additional analytes tested, 10 showed no difference between treatment conditions (Supplemental Figure 10) and 3 were undetectable (IL-1 $\beta$, IL-7, IL-15, data not shown). Overall, these data demonstrate that HIV-specific mAbs that do not prevent HIV from engaging CD4 on pDCs can specifically enhance the IFN response to the virus.

Enhancement by HIV-Ab complexes requires receptor-mediated binding and IRF7/NF- $K B$-signaling pathways. To further understand how HIV-Ab complexes enhanced the pDC IFN response compared with HIV alone, we reexamined the role of pathways defined as critical for pDC sensing of HIV in the absence of Ab. Culturing pDCs in the presence of CD4-blocking mAbs or the CCR5 inhibitor MVC not only negated Ab-mediated enhance- ment by mAb 4E10, but completely blocked all IFN production, demonstrating that $\mathrm{Ab}$ enhancement of IFN production does not circumvent the requirement for CD4 and coreceptor engagement (Figure 5A). Additionally, we saw that later stages of the HIV life cycle were not required for $\mathrm{mAb} 4 \mathrm{E} 10$ enhancement, since inhibition of reverse transcription (FTC) or integration (RAL) did not diminish $\mathrm{Ab}$ enhancement (Figure $5 \mathrm{~B}$ ). To determine whether the downstream signaling cascades were altered, we assessed dependence of the enhanced IFN response with 4 E10 on IRF7 phosphorylation and $\mathrm{NF}-\kappa \mathrm{B}$ and $\mathrm{p} 38$ MAPK signaling. We saw equivalent IRF7, IRF5, p-IRF7, p65, p38, and JNK levels in response to HIV in the presence and absence of enhancing $\mathrm{mAb}$ (Supplemental Figure 11, A and B). Additionally, inhibition of NF- $\kappa$ B or p38 MAPK signaling led to complete suppression of IFN production whether or not enhancing mAbs were present (Figure $5 \mathrm{C}$ ). It has been previously shown that HIV does not activate pDCs as robustly as TLR7 agonists or other viral infections (23). Activated pDCs are better producers of IFN, so we sought to determine whether the addition of HIV-Ab complexes altered the activation state of the pDCs, making them more potent effectors of the innate immune system. To test whether the addition of HIV-Ab complexes altered pDC activation, we assessed HLA-DR and CD86 expression on the cells with and without 4 E10 treatment. We saw that the addition of HIV-4E10 complex did not enhance HLA-DR or CD86 expression when compared with HIV alone; however, the addition of the TLR7 agonist resiquimod did increase HLA-DR and CD86 expression levels (Supplemental Figure 12). In sum, our data show that HIV-induced pDC IFN production in the presence of enhancing mAbs still requires HIV engagement of CD4CCR5 and that enhancing $\mathrm{mAbs}$ do not alter the downstream signaling pathways engaged or the activation state of the pDCs.

$A b$ enhancement of IFN production circumvents fusion and requires $F c \gamma R$ engagement, with signaling through TLR 7 and TLR9, which is absent with HIV alone. We initially hypothesized 

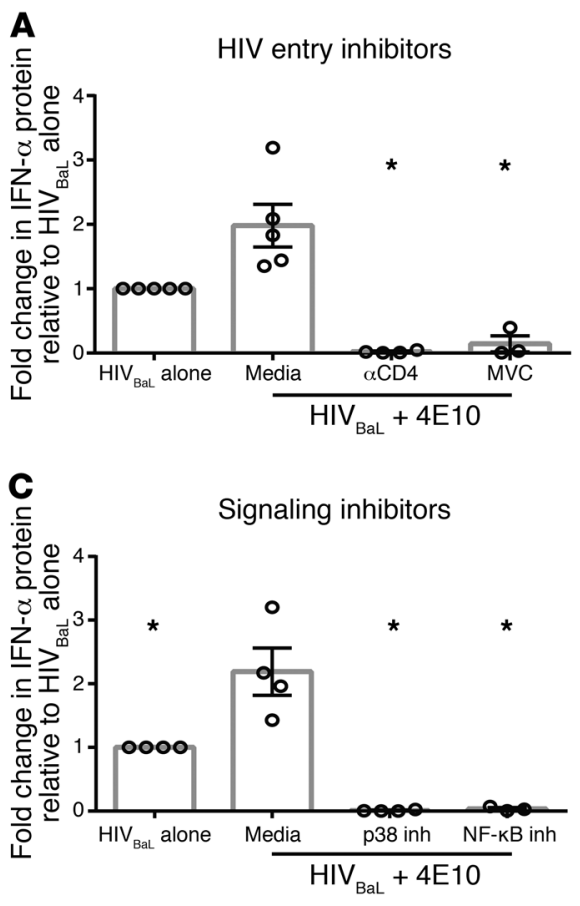

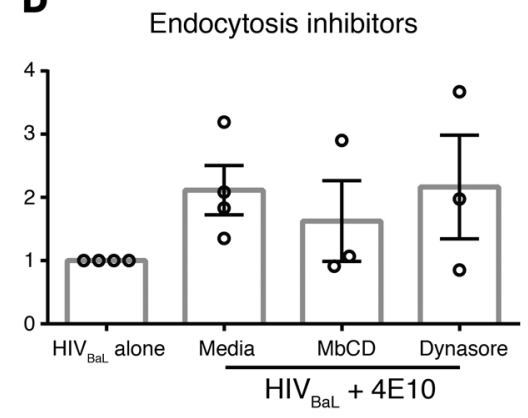

B

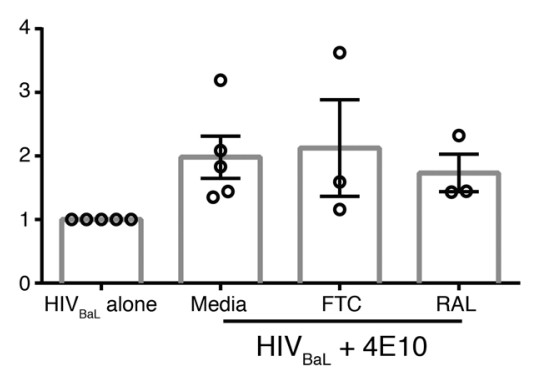

D
Figure 5. IFN enhancement by HIV-specific Abs requires CD4 and CCR5 binding and IRF7/NF- $K B$ signaling, but not endocytosis or active replication. Human pDCs were cultured with (A) HIV entry inhibitors $(n=4)$, (B) HIV replication inhibitors $(n=3)$, (C) signaling inhibitors $(n=4)$, or $(\mathbf{D})$ endocytosis inhibitors $(n=3)$ for 1 hour, followed by the addition of HIV $_{\text {BaL }}$ with and without 4 E10 for 15 hours. Supernatants were harvested and assessed for IFN- $\alpha$ protein production. Each data point indicates the average IFN- $\alpha$ production from 1 donor's pDCs tested in at least duplicate and normalized to the media condition. Error bars and gray boxes represent the SEM and the mean, respectively. Conditions were compared using 1-way ANOVA with Dunnett's multiple comparisons test. ${ }^{*} P<0.01$. that gp41-specific Abs enabled endocytosis of HIV as well as receptor-mediated entry, increasing overall viral entry. To assess this, we again used M $\beta C D$ and Dynasore to prevent clathrin- and caveolar-mediated endocytosis, respectively. However, inhibiting endocytosis did not suppress the enhancing effect of gp41specific mAb 4E10 (Figure 5D), suggesting that enhancement isn't mediated by endocytosis of virions. Next, we assessed whether $4 \mathrm{E} 10$ enhancement of IFN production required membrane fusion. While adding the fusion inhibitor T20 significantly suppressed IFN production with HIV alone (Figure 1D), the level of IFN produced in response to HIV with enhancing 4E10 remained unchanged in the presence of T20 (Figure 6A). These data demonstrate that 4E10 mediates HIV entry into pDCs through a mechanism distinct from endocytosis or virion fusion with the cell membrane and negates dependence on fusion of virion with the pDC membrane for HIV sensing. We next determined whether this alternate method of signaling required $\mathrm{Fc} \gamma \mathrm{R}$ engagement. Initially, we confirmed that our purified pDCs expressed Fc $\gamma \mathrm{R} 2$ (CD32) after isolation $\left(30 \% \mathrm{CD}^{2}\right)$ and after HIV activation $\left(50 \% \mathrm{CD}^{2}\right)^{\text {) }}$ (Supplemental Figure 13). Blockade of all Fc $\gamma$ Rs with the commercially available Fc Block (BD Biosciences) alters the pDC IFN response to HIV in the absence of Abs and was thus not an effective way to assess $\mathrm{Fc} \gamma \mathrm{R}$ dependence (30). Therefore, we used 2 alternative methods to prevent binding of the enhancing $\mathrm{mAb} 4 \mathrm{E} 10$ to $\mathrm{Fc} \gamma \mathrm{Rs}$. The first method was PNGase treatment, which removed all N-linked glycans from 4E10 via digestion (Figure $6 \mathrm{~B}$, deg 4E10). The removal of glycans from mAbs prevents the $\mathrm{Fc}$ from binding to any $\mathrm{Fc} \gamma \mathrm{R}$, with no effect on the Fab portion of the Ab (31-33). To confirm that deglycosylation did not affect $\mathrm{mAb}$ binding to HIV, we compared the neutralization capacity of deglycosylated 4E10 (deg4E10) with that of intact 4E10 and observed no difference (Supplemental Figure 14). The second method employed to prevent $4 \mathrm{E} 10$ binding to Fc $\gamma$ Rs was steri- cally blocking Fc $\gamma \mathrm{R}$ engagement by pretreating the pDCs with a $\mathrm{mAb}$ that binds to, but does not induce, signaling through Fc $\gamma \mathrm{R} 2$ (34). In contrast with the higher IFN protein levels observed with $4 \mathrm{E} 10$, IFN production with either deg4E10 or with $4 \mathrm{E} 10$ sterically prohibited from Fc $\gamma$ R2 engagement was comparable to that of HIV cultured with pDCs alone (Figure 6B). This suggests that FcR engagement is required for the $\mathrm{mAb}$ to enhance $\mathrm{PDC}$ IFN production in response to HIV.

Additionally, we tested whether the presence of enhancing $\mathrm{mAb}$ altered TLR sensing of the virus. Inhibition of TLR7 signaling by a TLR7 antagonist led to complete suppression of IFN production with or without $4 \mathrm{E} 10$ present. However, inhibition of TLR9 signaling negated only enhancement of IFN production, returning IFN production to levels seen in the absence of mAb. This suggests that Ab-mediated enhancement of pDC IFN production occurs through TLR9 engagement of HIV in conjunction with TLR7 sensing (Figure 6C).

Abs isolated from subjects with chronic HIV infection enhance IFN production. We demonstrated in vitro that HIV-specific mAbs that blocked the interaction between gp120 and CD4 prevented pDC production of IFN, while Abs that bound the HIV envelope without blocking CD4 engagement largely enhanced IFN production. To assess the role of polyclonal Abs generated in vivo, we isolated IgG from serum collected at years 1 and 2 after seroconversion from 13 HIV-infected subjects in the Baltimore-Washington, DC, Center of the Multicenter AIDS Cohort Study (MACS) (http:// aidscohortstudy.org/). These early time points were chosen because CD4 counts were high in every subject, which avoided assessment of responses in the setting of profound immunosuppression. Specimens were collected prior to the availability of effective ART, so no subject was on ART at the time points assessed. Because prolonged high-level IFN production is associated with chronic infection and immune activation $(8,35)$, we 

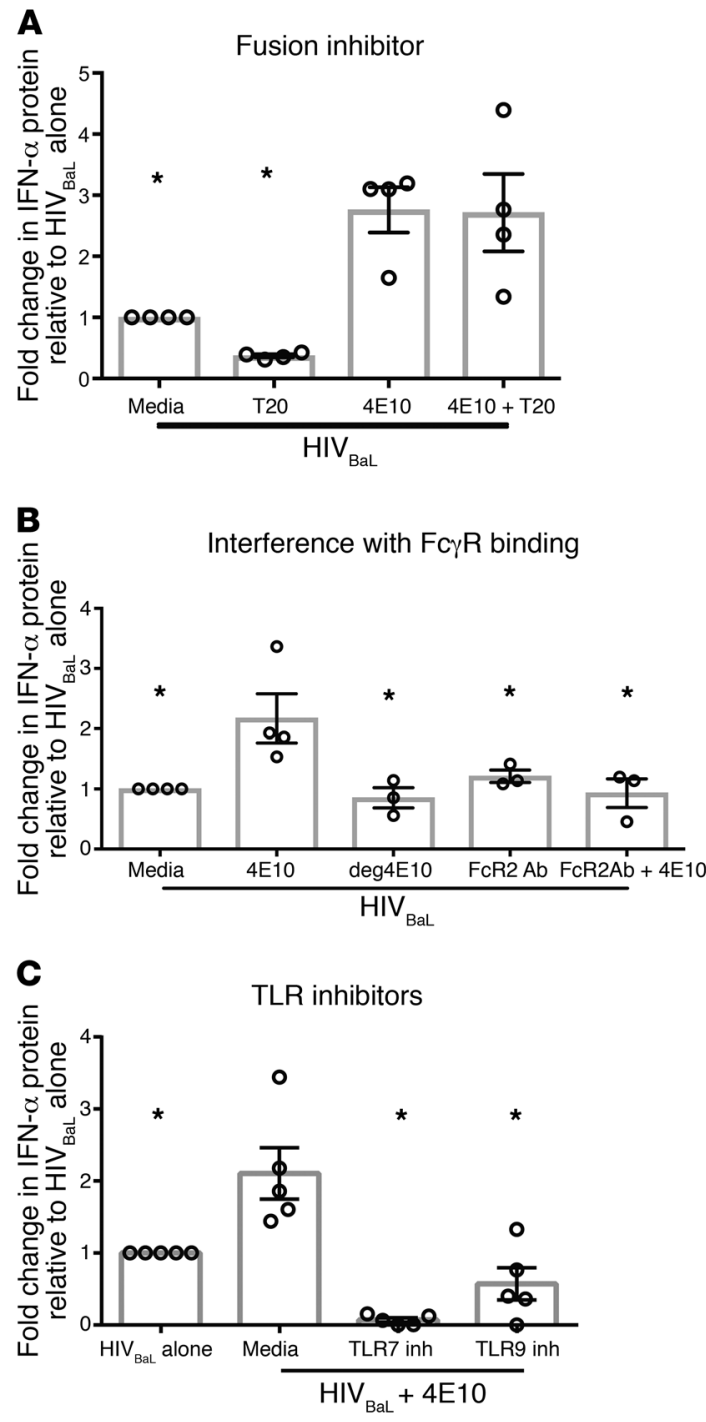

Figure 6. HIV-Ab complex enhancement of HIV sensing bypasses fusion, requires FcyR engagement, and signals through both TLR7 and TLR9 to increase IFN- $\alpha$ production. Human $\mathrm{pDCs}$ were cultured with (A) the HIV fusion inhibitor T20 $(n=4)$, (B) Fc $\gamma$ R2-blocking Ab $(n=3)$, or (C) TLR inhibitors $(n=5)$

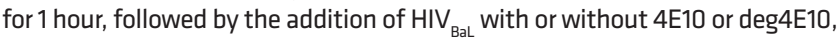
deglycosylated (B only) for 15 hours. Supernatants were harvested and assessed for IFN- $\alpha$ protein production. Each data point indicates the average IFN- $\alpha$ production from 1 donor's $\mathrm{pDC}$ s tested in at least duplicate and normalized to the media alone plus HIV $\mathrm{BaL}_{\text {L }}$ condition. Error bars and gray boxes represent the SEM and the mean, respectively. Conditions were compared using 1-way ANOVA with Dunnett's multiple comparisons test. ${ }^{*} P<0.05$.

hypothesized that IgG from HIV-infected subjects would enhance pDC IFN production in response to HIV. Given our previous in vitro data suggesting that $\mathrm{mAb}$ against gp120 suppress IFN production by pDC, we first tested the isolated IgG for gp120 binding in an ELISA. All IgG samples tested bound to HIV $_{\text {BaL }}$ gp120 lysate, though to varying degrees (Supplemental Figure 15). We next tested the IgG isolated from the 13 HIV-infected subjects for the ability to suppress or enhance pDC production of IFN in response to $\mathrm{HIV}_{\text {BaI }}$ in the same pDC assay used to assess the effects of 4E10. Despite some of the polyclonal IgG binding gp120, IgG isolated from 0 of the 13 subjects suppressed HIV $_{\text {BaL }}$-induced IFN production by pDC.
In contrast, polyclonal IgG isolated from 12 of the 13 subjects significantly enhanced pDC production of IFN in response to $\mathrm{HIV}_{\text {BaL }}$ compared with polyclonal IgG from HIV-uninfected subjects (Figure 7A). IgG isolated from subjects 1 year after HIV infection did not differ from that isolated 2 years after infection in the capacity of enhancing IFN production (Figure 7B). Consistent with gp120binding affinity not being the primary determinant of the effect on pDC IFN production, we saw no correlation between IFN enhancement and gp120 binding as measured by ELISA (Figure 7C).

We could not isolate enough autologous HIV from each subject to test the effect of IgG sensing of their own HIV. Therefore, we assessed the effect of naturally generated polyclonal IgG from the 13 subjects in early HIV infection to another HIV laboratory stain, $\mathrm{HIV}_{\text {IIIB }}$, to determine whether the results with $\mathrm{HIV}_{\text {BaL }}$ were representative of the subjects' response to HIV. Nearly identical results were seen when the IgG specimens were added to HIV $_{\text {IIIB }}$ prior to culture with pDCs. All 13 IgG specimens from HIVinfected subjects enhanced $\mathrm{HIV}_{\mathrm{IIIB}}$-induced IFN production, with no significant differences between IgG isolated from year 1 and year 2 after seroconversion in the capacity to enhance $\mathrm{pDC}$ production of IFN (Figure 7, D and E). Additionally, as with the $\mathrm{HIV}_{\text {BaL }}$ strain, we saw no correlation between IFN enhancement and gp120 binding, as measured by ELISA (Figure 7F). The degree and rank order of enhancement were similar, but not identical, between the $2 \mathrm{HIV}$ strains. These data suggest that the effects on HIV sensing by pDC of IgG generated in HIV-infected people are similar regardless of HIV strain used to stimulate pDCs and that IFN enhancement occurs with Abs generated in natural human infection, not just with mAbs selected for potent HIV-binding capacity.

\section{Discussion}

Although IFNs are essential in the resolution of acute viral infections, they have been associated with enhanced immune activation in chronic HIV and SIV infection as well as in other chronic infections $(4,8,35)$. Identifying the cause of persistent, high-level IFN responses during chronic HIV infection is important in understanding its pathogenesis. This study demonstrates a potential mechanism for enhanced IFN production after the acute phase of HIV infection at times when $\mathrm{Ab}$ responses to HIV are present. We have shown that $\mathrm{HIV}_{\mathrm{BaL}}$ stimulation of pDCs to produce IFN requires receptor-mediated entry, envelope fusion, viral uncoating, and engagement of TLR7 with defined downstream signaling that involves NF- $\mathrm{kB}$, JNK, and p38 MAPK (Figure $8 \mathrm{~A}$ ). Previous studies have shown that CD4 binds HIV and that TLR7 is engaged in pDCs to sense HIV and produce IFN $(22,23)$. However, ours is the first assessment, to our knowledge, of dependence on CCR5 engagement and of the stages of the complete HIV life cycle required to effect pDC sensing of HIV. In addition, previous studies attributed pDC activation to endocytosis of HIV because they observed loss of IFN- $\alpha$ production when acidification inhibitors were present in the culture (16). However, endosomal TLRs require acidification to signal, so acidification inhibitors block TLR signaling regardless of the mechanism of virus entry (36). By inhibiting endocytosis without blocking the acidification process critical to TLR signaling, we have shown that alternative methods of viral uptake activate the IFN pathway in $\mathrm{pDCs}$, not endocytosis. 
A
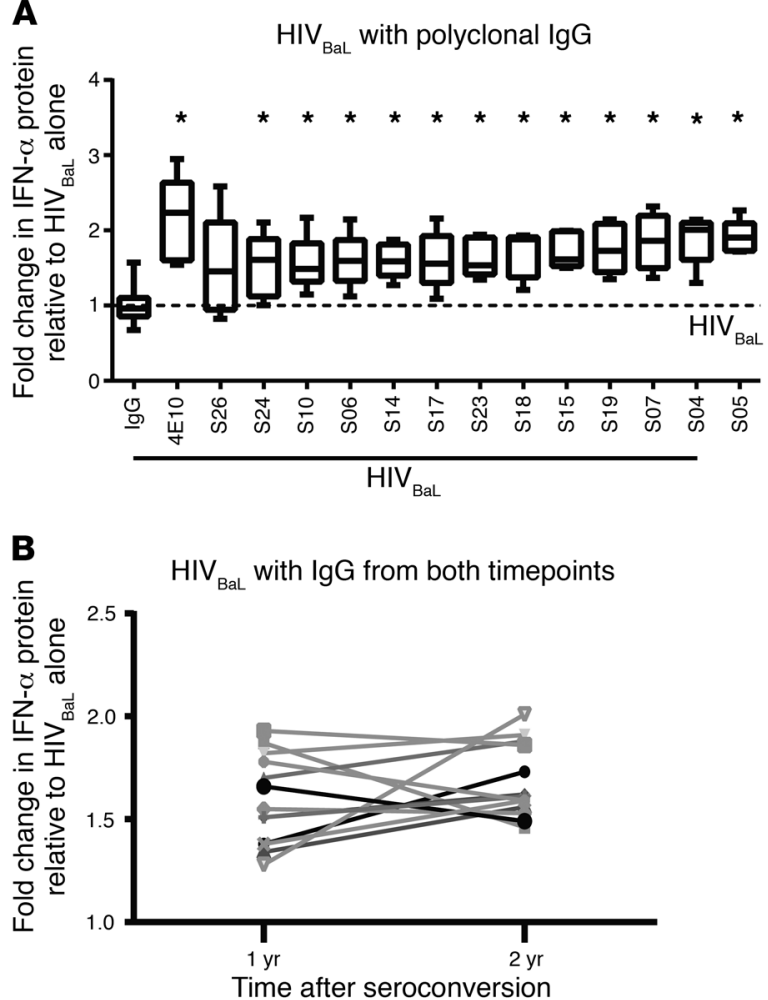

C gp120 binding vs. IFN enhancement

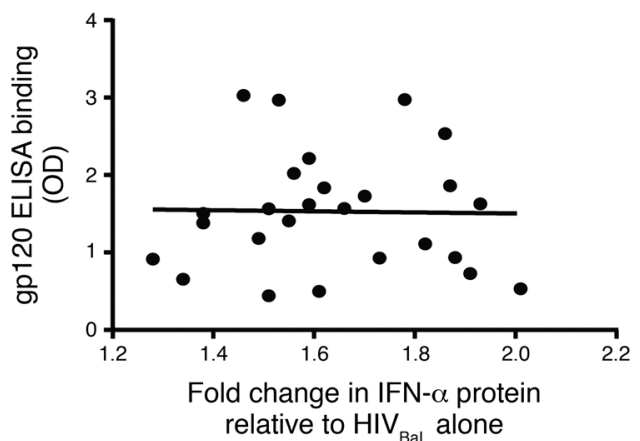

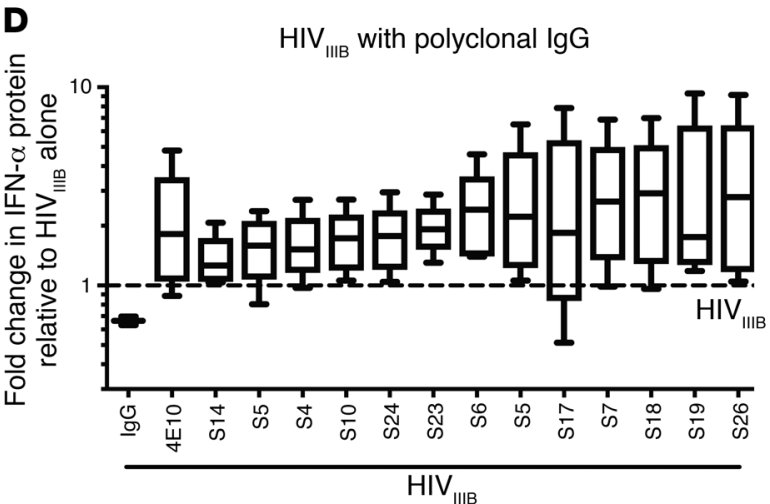

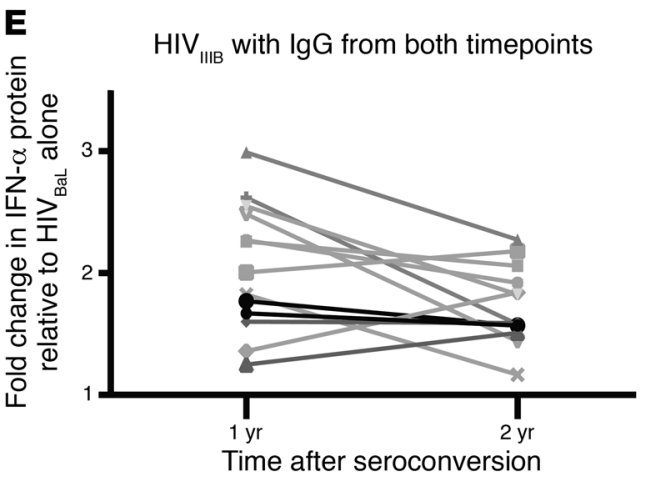

$\mathbf{F}$

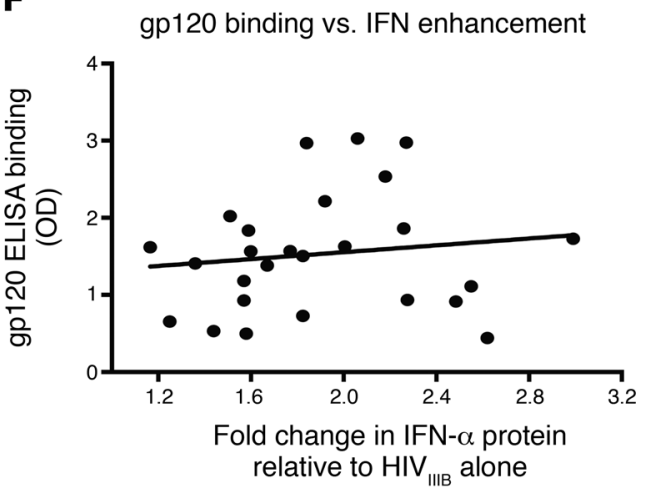

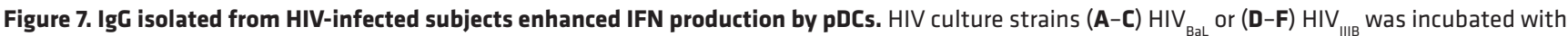
polyclonal IgG isolated from 13 HIV-infected subjects at either 1 or 2 years after seroconversion for 1 to 2 hours and then added to pDCs from at least 5 distinct donors. Supernatants were harvested after 15 hours and assessed for IFN- $\alpha$. (A and D) IgG isolated from 2 years after infection for 13 subjects ordered

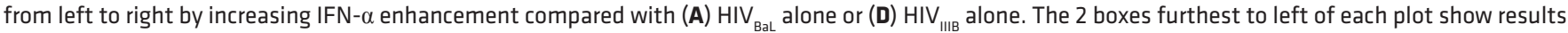
with polyclonal IgG isolated from HIV-uninfected subjects and 4E10, as negative and positive controls, respectively. Box plots indicate the median, the $75 \%$ and $25 \%$ quartiles, and the $95 \%$ and $5 \%$ outliers $(n=5)$. All samples were normalized to the HIV-alone conditions indicated by the dashed lines. Conditions were compared using 1-way ANOVA with Dunnett's multiple comparisons test. ${ }^{*} P<0.05$. (B and E) Comparison of IFN- $\alpha$ enhancement by IgG isolated from 1 and 2 years after infection relative to (B) HIV $_{\text {Bat }}$ alone or (E) HIV $\mathrm{HIB}_{\text {a }}$ alone. (C and $\mathbf{F}$ ) Correlation of gp120 binding versus enhancement of IFN- $\alpha$ by polyclonal IgG from all subjects relative to (C) HIV $_{\text {BaL }}$ alone or (F) HIV $_{\text {IIIB }}$ alone.

Once HIV enters the pDC, there are multiple methods through which viral RNA could access TLR7 in the endosome. Previous studies have shown that autophagy is required for IFN production in viral sensing by pDC, suggesting that the autophagy pathway connects viral RNA in the cytosol and TLR7 engagement within the lysosome $(37,38)$. It has been shown previously that TLR7 signaling within the lysosome leads to NF-KB and IRF7 signaling, while TLR7 signaling within the endosome leads primarily to IRF7 without NF- $\mathrm{KB}$ signaling (22). Thus, our data showing that HIVinduced IFN production after TLR7 engagement requires NF- $\mathrm{kB}$ signaling as well as IRF7 activation support pDC autophagy and TLR signaling in the lysosome rather than in the endosome. Similarly, signaling in the lysosome rather than the endosome is supported by our demonstration that endocytosis inhibition has no effect on HIV sensing by pDCs. We attempted to test the autophagy pathway, but were unable to find specific autophagy inhibitors that had no effect on TLR7 signaling. In sum, our data demonstrate for what we believe is the first time that HIV is sensed by pDCs through receptor-mediated entry dependent on CCR5 coreceptor engagement, envelope fusion, viral uncoating, and TLR7 
A

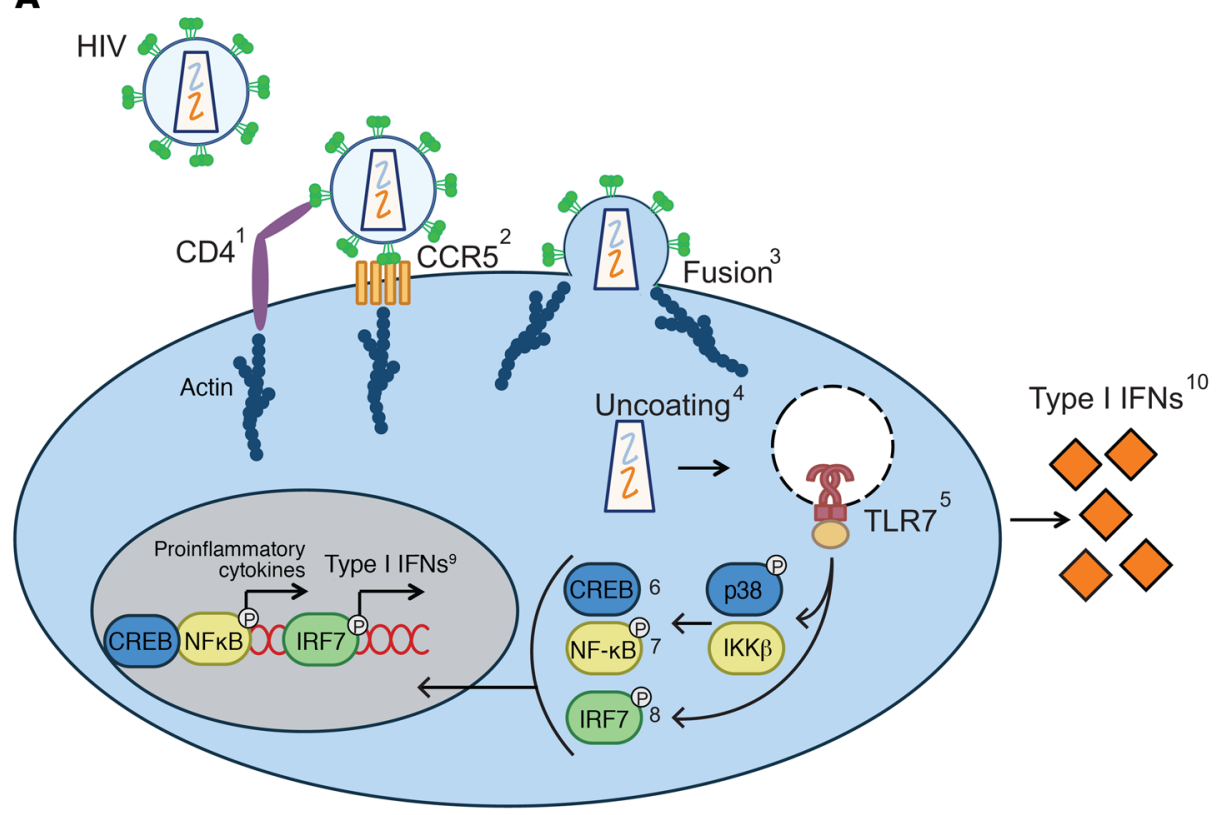

B

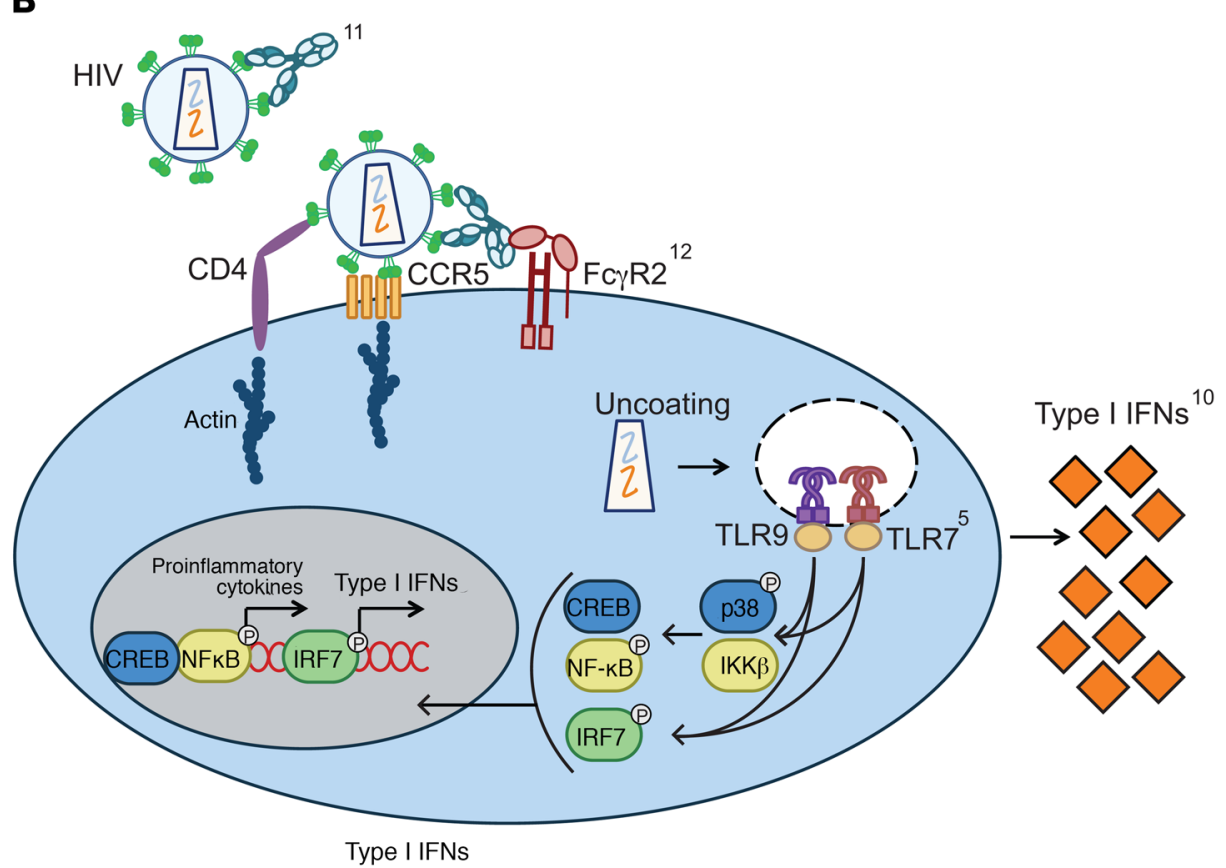

Figure 8. Suggested mechanism by which HIV-specific Abs that don't block CD4 binding enhance type I IFN-s. Representative diagrams of (A) IFN production by pDCs exposed to HIV and (B) enhanced IFN production by pDCs exposed to HIV preincubated with envelope-specific Abs that do not block CD4 binding. The 2 conditions diverge at 2 steps. First, the addition of Abs allows sensing of HIV without membrane fusion and through engagement of the $\mathrm{F} c \gamma \mathrm{R} 2 \mathrm{~A}$; however, the engagement of both CD4 and CCR5 is still required. Secondly, in the presence of $A b$, both endosomal TLRs, 7 and 9, sense the virus, while TLR9 plays no role in the absence of $A b$. Inhibitors, Abs, and/or methods used to elucidate each step of the mechanism are listed here and numbered in the diagrams ( $A$ and $\mathbf{B}$ ). Only steps of HIV sensing that differ when Ab is present are numbered in B. 1. anti-human CD4 Ab; 2. Maraviroc; 3. Enfuvirtide (T20); 4. CypA; 5. TLR 7 and TLR9 oligo inhibitors; 6. MAPK p38 inhibitor and Phosflow detection of phosphorylated p38; 7. IKK $\beta$ inhibitor and Phosflow detection of phosphorylated NF-kB p65; 8. Phosflow detection of $p-I R F 7$ 9. Quantitative reverse-transcriptase PCR (RTqPCR) detection of type I IFN mRNA; 10. ELISA detection of type I IFN protein. The following are shown in B only: 11. gp120 and gp41 mAbs and polyclonal IgG; 12 . VRC01 variants, deglycosylated Abs, and FcR2-blocking Abs. engagement with downstream signaling via p65, JNK, and p38 MAPK without dependence on stages of the HIV life cycle after uncoating or on endocytosis.

To test the hypothesis that Abs modulate IFN production in persistent infection, we assessed the effect of HIV-specific Abs on IFN production by pDCs. We demonstrated that mAbs that interfere with HIV gp120-CD4 binding suppress the IFN response independently of their ability to bind Fc $\gamma$ R. These data agree with previous studies showing that the gp120-CD4 interaction is critical for IFN production by pDCs $(16,17,23,34)$. However, we have also demonstrated that a subset of HIV-specific Abs that do not interfere with CD4 binding substantially enhanced the IFN response. Using a panel of gp41-specific mAbs, we determined that neither the specific gp41 epitope targeted nor the mAb's ability to neutralize HIV infection of CD4 T cells determined the capacity to enhance pDC IFN production. Ab-mediated enhancement did not require receptor-mediated fusion, but did require Fc $\gamma$ R engagement and both TLR7 and TLR9, with TLR9 signaling unique to HIV sensing in the presence of enhancing mAbs (Figure $8 \mathrm{~B})$. Enhanced pDC IFN production with HIV-Ab complexes versus HIV alone has not been previously shown and represents a mechanism for IFN regulation in chronic infection that may in part explain the increased IFN response and immune activation observed in chronic HIV.

We extended our findings with $\mathrm{mAb}$ by demonstrating that polyclonal IgG isolated from untreated HIV-infected patients also 
enhanced pDC IFN production in response to 2 different laboratory strains of HIV. This enhancement was not due to a lack of gp120-specific Abs because all of the polyclonal IgG samples had some gp120-binding activity. Thus, in contrast with previous studies showing that anti-HIV envelope mAbs suppress pDC sensing of virus, Abs generated in vivo enhanced IFN production from $\mathrm{pDCs}$ in response to HIV $(16,34)$. These data suggest that Ab-mediated enhancement of IFN production by pDCs may be more relevant in vivo than the previously described $\mathrm{mAb}$ suppression in vitro through blocking HIV engagement of pDC CD4. A limitation of this study is that we did not study IgG isolated from people with virologically suppressed HIV, in whom some studies have shown continued immune activation (39). We did not study IgG from those subjects because our in vitro conditions do not mimic the intermittent bursts of viremia with activation of the latent reservoir that characterize treatment-induced virologic suppression. An additional limitation of this study is that we could not isolate autologous HIV in sufficient quantities from HIV-infected subjects to test with pDCs isolated from 5 different donors. However, we did test 2 distinct HIV culture strains with different tropism and distinct capacity to activate pDCs and observed similar enhancing effects of polyclonal IgG from HIV-infected subjects with both. This suggests that the effects were not specific to one HIV strain. The fact that Abs isolated from HIV-infected patients enhanced IFN production in response to 2 different culture strains of HIV suggests that a humoral response to HIV that fails to control infection may lead to enhanced immune activation, including increased ISG expression and higher frequencies of $\mathrm{CD}^{+}$and $\mathrm{CD}^{+} \mathrm{T}$ cells expressing activation/proliferation markers. A final limitation of this study is that we do not know the sequence of the FCRG2A gene for each healthy donor tested. It had been reported that a polymorphism (H131R) found in the extracellular domain of FCGR2A can alter responses to various pathogens, increase the chance of developing autoimmune diseases, and alter response to mAb treatments (4043). The WT sequence $(\mathrm{HH})$ is thought to allow for better antibodydependent cell-mediated cytotoxicity (ADCC) on NK cells and may also contribute to better uptake of pathogens in other innate cells that express the FC $\gamma \mathrm{R} 2 \mathrm{~A}$ receptor, such as pDCs (41). Knowledge of the FCGR2A sequence for each healthy donor used in this work may have helped to explain the variability seen in IFN- $\alpha$ production in our assay. However, these polymorphisms are not known to have an effect on HIV control and disease progression.

Although not shown previously in chronic infection, pDC-Ab modulation of IFN has been demonstrated with pDCs and large self-DNA-Ab complexes responsible for the IFN signature seen in autoimmune diseases, such as systemic lupus erythematosus (44, 45). It has been shown that IFN produced by pDCs causes apoptosis of the pDCs secreting the IFN and of pDCs in the surrounding environment (46). Thus, in an infection that can be controlled by the adaptive immune system, high-level IFN may silence the pDC response as the adaptive immune system clears that infection. In contrast, in HIV, the immune system cannot remove all of the antigen and continued immune complex formation may persistently stimulate new pDC IFN production. Our data suggest that Abs produced in vivo in untreated HIV infection could contribute to persistent high-level IFN responses during chronic HIV infection, representing what we believe to be a novel mechanism of immune activation. Vaccination or other strategies that enhance the humoral response to HIV gp120 at the CD4-binding site relative to binding of other HIV envelope epitopes may have therapeutic potential in reducing immune activation. In addition, mAbs directed against HIV are being tested currently in clinical trials to prevent HIV rebound following discontinuation of ART and are being considered in HIV prevention trials (47-50). Our data demonstrate that the effects of $\mathrm{mAb}$ on $\mathrm{pDC}$ production of IFN should be considered prior to $\mathrm{mAb}$ selection for clinical trials because mAbs that enhance pDC production of IFN might increase immune activation. In sum, our data provide an explanation for high-level IFN production and immune activation in chronic HIV infection that should be considered in the selection of $\mathrm{mAbs}$ for clinical use.

\section{Methods}

Expansion of HIV cell culture strains, purification of HIV, and deactivation by $A T-2$ HIV $_{\mathrm{BaL}}$ and $\mathrm{HIV}_{\text {IIIB }}$ were obtained from Joel Blankson and from Suzanne Gartner (University of Maryland, College Park, Maryland, USA), respectively, and expanded as previously described (18). In brief, 10 to $20 \times 10^{6} \mathrm{PHA}$-activated CD4 ${ }^{+} \mathrm{T}$ cells were spinoculated $(1,200 \mathrm{~g}$, room temperature, 2 hours) with 0.5 to $1 \mu \mathrm{g}$ of HIV p24. Spinoculated cells were cultured for 10 days prior to harvest of infectious culture supernatant. Where indicated, experiments were complete with either infectious HIV supernatant or with purified HIV. HIV virus was purified via ultracentrifugation from infectious culture supernatant. Supernatants were layered on top of a $20 \%$ sucrose cushion and ultracentrifuged at $130,000 \mathrm{~g}$ for 2 hours at $4^{\circ} \mathrm{C}$. Virus pellets were then reconstituted with 1 to $2 \mathrm{ml}$ of RPMI plus $10 \%$ fetal bovine serum (Invitrogen) overnight at $4^{\circ} \mathrm{C}$. HIV p24 Ag was measured using the Alliance p24 ELISA Kit (Perkin Elmer) per the manufacturer's recommendations. AT-2-deactivated virus was produced as previously described (20). Briefly, HIV was treated with $300 \mu \mathrm{M}$ AT-2 (Sigma-Aldrich) for 1 hour at $37^{\circ} \mathrm{C}$. After incubation, the virus was filtered using a $100 \mathrm{kDa}$ Amicon Ultra $0.5 \mathrm{ml}$ filter (Millipore).

HIV mAbs. The following Abs were obtained from the AIDS Research and Reference Reagent Program, Division of AIDS, NIAID, NIH: anti-HIV-1 gp120 mAbs, IgG1 b12 (51-54) from Dennis Burton and Carlos Barbas (Scripps Institute, La Jolla, California, USA); PG16 (55); PG9 (55); and $2 \mathrm{G} 12$ (28, 56-59) from Hermann Katinger (Polymun Scientific, Klosterneuburg, Austria); anti-HIV-1 gp41 mAbs 4E10 (60), 2F5 (28, 61, 62), and 5F3 (28) from Hermann Katinger; 246-D (63-66) from Susan Zolla-Pazner (Mount Sinai, New York, New York, USA). 4E10 mAb was also purchased from Polymun Scientific. Gp120 mAb VRC01 and VRC01 FcR-binding variants were produced as previously described (27).

HIV-infected subject sample selection. Serum samples from HIVinfected subjects not on ART and in early infection were obtained from the Baltimore-Washington, DC, site of MACS. MACS is an ongoing prospective cohort study of the natural and treated history of HIV infection in men who have sex with men at 4 sites in the US (Baltimore, Maryland/Washington, DC; Chicago, Illinois; Los Angeles, California; and Pittsburgh, Pennsylvania) $(67,68)$. MACS participants are followed with semi-annual study visits that include questionnaires, a brief physical exam, and phlebotomy for laboratory tests and storage of serum, plasma, and peripheral blood mononuclear cells. HIVuninfected participants are tested at each visit for HIV seroconversion. 
Two frozen plasma samples were obtained from all men 1 and 2 years after the estimated time of seroconversion, which was taken as the midpoint between the last seronegative visit and the first seropositive visit.

pDC purification and activation. Freshly collected deidentified human blood Leuko Paks were obtained from the Johns Hopkins Blood Donor Center and the Anne Arundel Medical Blood Donor Center (Anne Arundel, Maryland, USA). PBMCs were isolated by Ficoll-Hypaque gradient centrifugation. pDCs were magnetically sorted by negative isolation per the manufacturer's protocol (Miltenyi Biotec) and cultured in RPMI 1640 media (Invitrogen), 10\% heat-inactivated pooled human AB serum (Atlanta Biological), and $1 \%$ MEM nonessential amino acids, sodium pyruvate, HEPES, and L-glutamine (Life Technologies). Postisolation pDCs were plated at $0.5-1 \times 10^{6}$ cells $/ \mathrm{ml}$ and cultured with $20-40 \mathrm{ng} / \mathrm{ml}$ of HIV p24, 0.02-0.1 $\mu \mathrm{M}$ resiquimod, a TLR7 agonist or ODN2216, and a TLR9 agonist (InvivoGen). For Ab experiments, HIV was preincubated for 1 to 2 hours at $37^{\circ} \mathrm{C}$ with $5 \mu \mathrm{g} / \mathrm{ml}$ of designated $\mathrm{mAb}$ or isolated polyclonal IgG to form immune complexes. The mixture was then added to pDC cultures, and IFN- $\alpha$ production was measured after 15 hours.

Quantitative real-time PCR. RNA was isolated with the RNeasy Kit (QIAGEN) and used to synthesize cDNA with the Superscript III Reverse Transcriptase System (Invitrogen). Quantitative PCR was performed with IDT predesigned primer/probe mixes using Light Cycler 480 Probes Master Mix (Roche). GAPDH and RPL13A were used as housekeeping controls. The ratio of target gene mRNA copies relative to the average mRNA copies from housekeeping controls was defined as $\Delta$. Ratio of normalized mRNA copies among experimental conditions was defined as $\Delta / \Delta$ and used to calculate the relative effect of HIV activation in pDCs.

IFN- $\alpha$ and additional analyte measurements. Human IFN- $\alpha$ was measured with the Human IFN- $\alpha$ Tissue Culture Kit from Meso Scale Discovery (MSD). Samples were tested per the manufacture's protocol using 25 $\mu \mathrm{l}$ of undiluted culture supernatant. The MSD multiplex cytokine, proinflammatory, and chemokine electrochemiluminescence immunoassays were used to assess 16 additional analytes (TNF- $\alpha$, IP10, MIP1 $\beta$, IL-6, IL-12/23p40, IL-10, IFN- $\gamma$, IL-16, IL-2, IL-8, Eotaxin, MCP1, MIP1 $\alpha$, IL-1 $\beta$, IL-15, IL-17). The assays were performed per the manufacturer's recommendations. Data were acquired on a SECTOR Imager SI2400. Results were analyzed using MSD Workbench software.

Modulation of IFN- $\alpha$ by inhibition of HIV entry or infection. Freshly isolated pDCs were preincubated with one of the following Abs or inhibitors for 1 hour at $37^{\circ} \mathrm{C}: 1.25 \mu \mathrm{g} / \mathrm{ml}$ anti-human CD4 mouse mAb (clone SK3, BioLegend), $1.25 \mu \mathrm{g} / \mathrm{ml}$ isotype control (clone MOPC-21, BioLegend), $10 \mu \mathrm{M}$ CCR5 receptor agonist Maraviroc (MVC, Selleckchem), $1 \mu \mathrm{M}$ fusion inhibitor Enfavirtide (T20, Sigma-Aldrich), $1.5 \mathrm{nM}$ CypA (Millipore), $1 \mu \mathrm{M}$ reverse transcriptase inhibitor FTC (Selleckchem), and $1 \mu \mathrm{M}$ integrase inhibitor RAL (Selleckchem). Virus with or without $\mathrm{HIV} \mathrm{Ab}$ was then added to the pDCs and IFN- $\alpha$ production measured after 15 hours.

Modulation of IFN by endocytosis, signaling, and TLR inhibitors. Freshly isolated pDCs were incubated with $20 \mu \mathrm{M}$ methyl- $\beta$-cyclodextrin (M $\beta C D)$ (Sigma-Aldrich), 1 hour at $37^{\circ} \mathrm{C}$, clathrin-mediated endocytosis inhibitor, the dynamin inhibitor Dynasore $80 \mu \mathrm{M}$ (Abcam); $1 \mu \mathrm{M}$ of IKK $\beta$ inhibitor, which inhibits NF- $\mathrm{BB}$ signaling, PF184 (Torcis); or $1 \mu \mathrm{M}$ of MAPK p38 inhibitor SB203580 (Cell Signaling Technology). TLR7 and TLR9 inhibitors have been previously described and were synthesized by IDT (69). Virus with and without HIV Ab was then added to the pDCs and IFN- $\alpha$ production measured after 15 hours.
Determining Fc $\gamma R$ usage. Fc $\gamma \mathrm{R}$ usage was determined after altering $\mathrm{Fc}$ binding in 3 ways. First, Fc binding was changed using previously described (27). In brief, the Fc domain of VRC01 Ab was point mutated to alter the Ab's affinity for different Fc $\gamma$ Rs. VRC01 WT maintains all WT amino acids and the strongest binding to Fc $\gamma$ R1. The Fc $\gamma$ R2A Ab contains a point mutation at position 236 from a glycine to an alanine, which confers strongest binding to the $2 \mathrm{~A}$ receptor. The Fc $\gamma \mathrm{R} 2 \mathrm{~B}$ Ab contains 2 point mutations, 1 at position 239, a serine to an aspartic acid, and 1 at position 332 , an isoleucine to a glutamic acid, both of which confer strongest binding to the $2 \mathrm{~B}$ receptor. The $\mathrm{Fc} \gamma \mathrm{R} 3 \mathrm{~A} \mathrm{Ab}$ contains 3 point mutations; the first 2 are present in the Fc $\gamma \mathrm{R} 2 \mathrm{~B} \mathrm{Ab}, \mathrm{S} 239 \mathrm{D}$, and I332E, the third mutation is at position 330, an alanine to leucine, all 3 of which confer strongest binding to the Fc $\gamma$ R3A receptor. Finally, the no-binding Ab has a point mutation at the N-linked glycosylation site, position 237 , an asparagine to glutamine, which prevents binding to all Fc $\gamma$ Rs. These mutations have no effect on the affinity or avidity of the Fab domain of the VRCO1 Ab. The second method used was Ab deglycosylation. Abs were digested using the nondenaturing protocol and the PNGase-F Remove-iT enzyme (New England BioLabs). In brief, $20 \mu \mathrm{g}$ of $4 \mathrm{E} 10$ was added to GlycoBuffer 2 and $\mathrm{H}_{2} \mathrm{O}$ to make a $1 \times 20 \mu \mathrm{l}$ reaction; the mixture was incubated at $37^{\circ} \mathrm{C}$ overnight. The PNGase-F enzyme was then removed using Chitin Magnetic beads (New England BioLabs) per the manufacturer's recommendations. The third method utilized a polyclonal Ab against Fc $\gamma \mathrm{R} 2$ to sterically block interactions as previously described (34). In brief, after isolation, pDCs were cultured with $10 \mu \mathrm{g} / \mathrm{ml}$ of goat anti-human Fc $\gamma$ R2 pAb (R\&D Systems) and incubated at $37^{\circ} \mathrm{C}$ for 1 hour. Virus with and without HIV Ab was then added to the pDCs and IFN- $\alpha$ production measured after 15 hours. Cells were stained with a PE mouse anti-human CD32 (BD Biosciences) to confirm that FcR2 was blocked during the course of the experiment.

Cell purity, $F c \gamma R$ expression, and protein phosphorylation staining by flow cytometry. After sorting, pDC purity was determined by flow cytometry using known pDC markers PE-Cy7 mouse anti-CD123 (BD Biosciences, catalog 560826), BV421 mouse anti-CD303, and PE mouse antiCD4 (BioLegend, catalog 5354212 and 344606). The pDC cultures were 95\% pure based on CD303, CD123, and CD4 expression. Cell activation marker expression was determined using PerCP-Cy5.5 mouse antiHLA-DR and APC mouse anti-CD86 (BD Biosciences, catalog 560652 and 555660). Fc $\gamma \mathrm{R}$ expression was determined using a PE mouse antiCD32 Ab (BD, catalog 552884). Intercellular IRF5 and IRF7 staining was completed using the following Abs: unconjugated rabbit anti-IRF5 (EPR6094, Abcam), donkey anti-rabbit AF488 secondary, AF647 mouse anti-IRF7 (BioLegend, catalog 656008), and the BD Cytofix/Cytoperm Kit per the manufacturers' instructions. In brief, poststimulation supernatants were removed and cell pellets were washed with $1 \times$ BD Perm/ Wash Buffer. Cells were then fixed and permeabilized with $50 \mu \mathrm{l}$ of cold BD Fixation/Permeabilization solution at $4^{\circ} \mathrm{C}$ for 20 minutes. Cells were then washed with BD Perm/Wash Buffer and stained with a panel of intercellular Abs per the manufacturer's instructions. The Abs used to detect phosphorylation were AF647 mouse anti-IRF7 (pS477/pS479), PE-Cy7 mouse anti-NF- $\kappa$ B p65 (pS529), PE-CF594 mouse anti-p38 MAPK (pT180/pY182), PE mouse anti-JNK (pT183/pY185), and BV421 mouse anti-STAT1 (pY701) (BD Biosciences, catalog 558630, 560335, $612565,562480,562985)$. To assess whether the above signaling molecules were phosphorylated in response to HIV activation, we used the Phosflow Kit and protocol developed by BD. In brief, poststimulation supernatants were removed and cell pellets were incubated with $100 \mu \mathrm{l}$ of warmed BD Cytofix Buffer for 10 minutes at $37^{\circ} \mathrm{C}$. Cells were then 
washed 2 times with 1\% BSA in 1× PBS, permeabilized by slowly adding $100 \mu \mathrm{l}$ of cold BD Phosflow Perm Buffer III, and incubated on ice at $-20^{\circ} \mathrm{C}$ for 30 minutes. Cells were washed 2 times with $1 \%$ BSA in $1 \times$ PBS and stained with the panel of Phosflow Abs above per the manufacturer's instructions. All data were acquired on a BD LSRII flow cytometer.

Isolation of polyclonal IgG from plasma samples. Polyclonal IgG (pAb) was isolated from plasma samples using protein A columns (Thermo Fisher Scientific) per the manufacturer's instructions. Isolated IgG was neutralized with binding buffer concentrated on 50 $\mathrm{kDa}$ Amicon Ultra $5 \mathrm{ml}$ filter (Millipore), and quantified using the Human IgG total ELISA Ready-SET-Go Kit (eBioscience) per the manufacturer's instructions.

HIV gp12O-binding ELISA. Polyclonal Ab binding to $\mathrm{HIV}_{\mathrm{BaL}}$-gp120 was quantified by ELISA as previously described (70). In brief, HEK293T cells were transfected with $\mathrm{HIV}_{\mathrm{Ba}}$-gp120-expressing plasmid and lysed 48 hours after transfection. Immulon $2 \mathrm{HB}$ flat-bottom strips (Thermo Fisher Scientific) were coated with $500 \mathrm{ng} / \mathrm{ml}$ Galanthus nivalis lectin (Sigma-Aldrich) and blocked with PBS containing 0.5\% Tween 20, $1 \%$ nonfat dry milk, and $1 \%$ goat serum for 1 hour at $37^{\circ} \mathrm{C}$. $\mathrm{HIV}_{\text {BaL }}$ gp12O cell lysates were added overnight at $4^{\circ} \mathrm{C}$. Binding of pAb was measured in 5-fold serial dilutions, starting at $125 \mu \mathrm{g} / \mathrm{ml}$. Binding was detected using HRP-conjugated anti-human IgG secondary Ab (BD Biosciences - Pharmingen).

Statistics. One-way ANOVA and 2-tailed paired $t$ tests were used to evaluate statistical significance of differences between groups. Differences were considered significant at $P<0.05$ or when otherwise indicated.

Study approval. All study protocols were approved by an IRB of the Johns Hopkins University School of Medicine. All adult subjects who provided blood samples also provided written informed consent, and parents or guardians of all participating children provided written informed consent on their behalf.

\section{Author contributions}

RTV, MAC, and ALC designed the project. RTV, ZTF, LKC, GM, and AT performed cellular biological experiments. JK and RTV performed gp120 ELISAs and IgG purifications. MEA and AWB provided VRC01 variants and thoughtful discussion of results. JNB provided thoughtful discussion and experimental advice. JBM provided the plasma samples from the BaltimoreWashington, DC, site of the MACS cohort and expertise in analysis of results and statistics. MAC and ALC provided expertise in design of experiments and analysis of results. RTV and ALC cowrote the paper. All authors edited the manuscript.

\section{Acknowledgments}

This work was supported by the National Institute of Allergy and Infectious Diseases (R01 AI108403, R01 AI102691, U01 AI35042, and K08 AI10269). Flow cytometry experiments were performed in the Becton Dickinson Immune Function Laboratory at the Johns Hopkins Bloomberg School of Public Health. The facility is supported in part by the Johns Hopkins University Center for AIDS Research (CFAR): 5P30AI094189-04 (Chaisson). LKC was supported by T32 GM007309 (NIH).

Address correspondence to: Andrea L. Cox, 855 N. Wolfe Street, Rangos Research Building, Suite 536, Baltimore, Maryland 21205, USA. Phone: 410.502.2715; Email: acox@jhmi.edu.
1. Bosinger SE, Utay NS. Type I interferon: understanding its role in HIV pathogenesis and therapy. Curr HIV/AIDS Rep. 2015;12(1):41-53.

2. Teijaro JR, et al. Persistent LCMV infection is controlled by blockade of type I interferon signaling. Science. 2013;340(6129):207-211.

3. Wilson EB, et al. Blockade of chronic type I interferon signaling to control persistent LCMV infection. Science. 2013;340(6129):202-207.

4. Sandler NG, et al. Type I interferon responses in rhesus macaques prevent SIV infection and slow disease progression. Nature. 2014;511(7511):601-605.

5. Li G, et al. Plasmacytoid dendritic cells suppress HIV-1 replication but contribute to HIV-1 induced immunopathogenesis in humanized mice. PLOS Pathog. 2014;10(7):e1004291.

6. Rotger M, et al. Comparative transcriptomics of extreme phenotypes of human HIV-1 infection and SIV infection in sooty mangabey and rhesus macaque. JClin Invest. 2011;121(6):2391-2400.

7. Farzadegan H, et al. Virologic and serologic markers of rapid progression to AIDS after HIV-1 seroconversion. J Acquir Immune Defic Syndr Hum Retrovirol. 1996;13(5):448-455.

8. Jacquelin B, et al. Nonpathogenic SIV infection of African green monkeys induces a strong but rapidly controlled type I IFN response. J Clin Invest. 2009;119(12):3544-3555.

9. Bosinger SE, et al. Global genomic analysis reveals rapid control of a robust innate response in SIV-infected sooty mangabeys. J Clin Invest. 2009;119 (12):3556-3572.

10. Zhen A, et al. Targeting type I interferon-mediated activation restores immune function in chronic HIV infection. J Clin Invest. 2017;127(1):260-268.

11. Cheng L, et al. Blocking type I interferon signaling enhances T cell recovery and reduces HIV-1 reservoirs. J Clin Invest. 2017;127 (1):269-279.

12. Lu LL, et al. A functional role for antibodies in tuberculosis. Cell. 2016;167 (2):433-443.e14.

13. Richter K, Oxenius A. Non-neutralizing antibodies protect from chronic LCMV infection independently of activating Fc $\gamma \mathrm{R}$ or complement. Eur J Immunol. 2013;43(9):2349-2360.

14. Rerks-Ngarm S, et al. Vaccination with ALVAC and AIDSVAX to prevent HIV-1 infection in Thailand. N Engl J Med. 2009;361 (23):2209-2220.

15. Barouch DH, et al. Vaccine protection against acquisition of neutralization-resistant SIV challenges in rhesus monkeys. Nature. 2012;482 (7383):89-93.

16. Beignon AS, et al. Endocytosis of HIV-1 activates plasmacytoid dendritic cells via Toll-like receptorviral RNA interactions. J Clin Invest. 2005;115 (11):3265-3275.

17. Pritschet K, et al. CD4- and dynamin-dependent endocytosis of HIV-1 into plasmacytoid dendritic cells. Virology. 2012;423 (2):152-164.

18. Chattergoon MA, et al. HIV and HCV activate the inflammasome in monocytes and macrophages via endosomal Toll-like receptors without induction of type 1 interferon. PLoS Pathog. 2014;10 (5):e1004082.

19. Campbell EM, Hope TJ. HIV-1 capsid: the multifaceted key player in HIV-1 infection. Nat Rev Microbiol. 2015;13(8):471-483.

20. Rossio JL, et al. Inactivation of human immunodeficiency virus type 1 infectivity with preservation of conformational and functional integrity of virion surface proteins. J Virol. 1998;72(10):79928001.

21. McKenna K, Beignon AS, Bhardwaj N. Plasmacytoid dendritic cells: linking innate and adaptive immunity. J Virol. 2005;79 (1):17-27.

22. O'Brien M, et al. Spatiotemporal trafficking of HIV in human plasmacytoid dendritic cells defines a persistently IFN- $\alpha$-producing and partially matured phenotype. J Clin Invest. 2011;121 (3):1088-1101.

23. O'Brien M, et al. CD4 receptor is a key determinant of divergent HIV-1 sensing by plasmacytoid dendritic Cells. PLoS Pathog. 2016;12(4):e1005553.

24. Stark GR, Darnell JE. The JAK-STAT pathway at twenty. Immunity. 2012;36(4):503-514.

25. Ivashkiv LB, Donlin LT. Regulation of type I interferon responses. Nat Rev Immunol. 2014;14(1):36-49.

26. Fitzgerald KA, et al. IKKepsilon and TBK1 are essential components of the IRF3 signaling pathway. Nat Immunol. 2003;4(5):491-496.

27. Boesch AW, et al. Highly parallel characterization of IgG Fc binding interactions. MAbs. 
2014;6(4):915-927.

28. Buchacher A, et al. Generation of human monoclonal antibodies against HIV-1 proteins; electrofusion and Epstein-Barr virus transformation for peripheral blood lymphocyte immortalization. AIDS Res Hum Retroviruses. 1994;10(4):359-369.

29. Moog C, et al. Protective effect of vaginal application of neutralizing and nonneutralizing inhibitory antibodies against vaginal SHIV challenge in macaques. Mucosal Immunol. 2014;7(1):46-56.

30. Green DS, Lum T, Green JA. IgG-derived Fc down-regulates virus-induced plasmacytoid dendritic cell (pDC) IFN alpha production. Cytokine. 2004;26(5):209-216.

31. Walker MR, Lund J, Thompson KM, Jefferis R. Aglycosylation of human IgG1 and IgG3 monoclonal antibodies can eliminate recognition by human cells expressing Fc gamma RI and/or Fc gamma RII receptors. Biochem J. 1989;259(2):347-353.

32. Nesspor TC, Raju TS, Chin CN, Vafa O, Brezski RJ. Avidity confers Fc $\gamma$ R binding and immune effector function to aglycosylated immunoglobulin G1. JMol Recognit. 2012;25(3):147-154.

33. Tao MH, Morrison SL. Studies of aglycosylated chimeric mouse-human IgG. Role of carbohydrate in the structure and effector functions mediated by the human IgG constant region. JImmunol. 1989;143(8):2595-2601.

34. Lederle A, et al. Neutralizing antibodies inhibit HIV-1 infection of plasmacytoid dendritic cells by an Fc $\gamma$ RIIa independent mechanism and do not diminish cytokines production. Sci Rep. 2014;4:5845.

35. Keir ME, et al. Generation of CD3+CD8low thymocytes in the HIV type 1-infected thymus. J Immunol. 2002;169(5):2788-2796.

36. Cao $\mathrm{H}$, et al. Innate immune response of human plasmacytoid dendritic cells to poxvirus infection is subverted by vaccinia $\mathrm{E} 3$ via its $\mathrm{Z}-\mathrm{DNA} / \mathrm{RNA}$ binding domain. PLoS ONE. 2012;7(5):e36823.

37. Lee HK, Lund JM, Ramanathan B, Mizushima $\mathrm{N}$, Iwasaki A. Autophagy-dependent viral recognition by plasmacytoid dendritic cells. Science. 2007;315(5817):1398-1401.

38. Zhou D, Kang KH, Spector SA. Production of interferon $\alpha$ by human immunodeficiency virus type 1 in human plasmacytoid dendritic cells is dependent on induction of autophagy. J Infect Dis. 2012;205(8):1258-1267.

39. Sauce D, et al. HIV disease progression despite suppression of viral replication is associated with exhaustion of lymphopoiesis. Blood. 2011;117(19):5142-5151.

40. Warmerdam PA, van de Winkel JG, Vlug A, Westerdaal NA, Capel PJ. A single amino acid in the second Ig-like domain of the human Fc gamma receptor II is critical for human IgG2 binding. J Immunol. 1991;147(4):1338-1343.

41. Musolino A, et al. Immunoglobulin G fragment $C$ receptor polymorphisms and clinical efficacy of trastuzumab-based therapy in patients with
HER-2/neu-positive metastatic breast cancer. J Clin Oncol. 2008;26(11):1789-1796.

42. Maiga B, et al. Fc gamma receptor IIa-H131R polymorphism and malaria susceptibility in sympatric ethnic groups, Fulani and Dogon of Mali. Scand J Immunol. 2014;79(1):43-50.

43. Zhang C, Wang W, Zhang H, Wei L, Guo S. Association of FCGR2A rs1801274 polymorphism with susceptibility to autoimmune diseases: A meta-analysis. Oncotarget. 2016;7(26):39436-39443.

44. Rönnblom L, Pascual V. The innate immune system in SLE: type I interferons and dendritic cells. Lupus. 2008;17(5):394-399.

45. Gilliet M, Cao W, Liu YJ. Plasmacytoid dendritic cells: sensing nucleic acids in viral infection and autoimmune diseases. Nat Rev Immunol. 2008;8(8):594-606.

46. Swiecki M, Wang Y, Vermi W, Gilfillan S, Schreiber RD, Colonna M. Type I interferon negatively controls plasmacytoid dendritic cell numbers in vivo. J Exp Med. 2011;208(12):2367-2374.

47. Scheid JF, et al. HIV-1 antibody 3BNC117 suppresses viral rebound in humans during treatment interruption. Nature. 2016;535(7613):556-560.

48. Bar KJ, et al. Effect of HIV antibody VRCO1 on viral rebound after treatment interruption. $N$ Engl JMed. 2016;375(21):2037-2050.

49. Lynch RM, et al. Virologic effects of broadly neutralizing antibody VRC01 administration during chronic HIV-1 infection. Sci Transl Med. 2015;7(319):319ra206.

50. Barouch DH, et al. Therapeutic efficacy of potent neutralizing HIV-1-specific monoclonal antibodies in SHIV-infected rhesus monkeys. Nature. 2013;503(7475):224-228.

51. Burton DR, Barbas CF, Persson MA, Koenig S, Chanock RM, Lerner RA. A large array of human monoclonal antibodies to type 1 human immunodeficiency virus from combinatorial libraries of asymptomatic seropositive individuals. Proc Natl Acad Sci U S A. 1991;88(22):10134-10137.

52. Barbas CF, et al. Recombinant human Fab fragments neutralize human type 1 immunodeficiency virus in vitro. Proc Natl Acad Sci U S A. 1992;89(19):9339-9343.

53. Burton DR, et al. Efficient neutralization of primary isolates of HIV-1 by a recombinant human monoclonal antibody. Science. 1994;266(5187):1024-1027.

54. Roben P, Moore JP, Thali M, Sodroski J, Barbas $\mathrm{CF}$, Burton DR. Recognition properties of a panel of human recombinant Fab fragments to the CD4 binding site of gp120 that show differing abilities to neutralize human immunodeficiency virus type 1. J Virol. 1994;68(8):4821-4828.

55. Walker LM, et al. Broad and potent neutralizing antibodies from an African donor reveal a new HIV-1 vaccine target. Science. 2009;326(5950):285-289.

56. Trkola A, et al. Human monoclonal antibody 2 G12 defines a distinctive neutralization epitope on the gp120 glycoprotein of human immunodeficiency virus type 1. J Virol. 1996;70(2):1100-1108.

57. Mascola JR, et al. Protection of macaques against pathogenic simian/human immunodeficiency virus $89.6 \mathrm{PD}$ by passive transfer of neutralizing antibodies. J Virol.1999;73(5):4009-4018.

58. Etemad-Moghadam B, Sun Y, Nicholson EK, Karlsson GB, Schenten D, Sodroski J. Determinants of neutralization resistance in the envelope glycoproteins of a simian-human immunodeficiency virus passaged in vivo. J Virol. 1999;73(10):8873-8879.

59. Crawford JM, et al. Characterization of primary isolate-like variants of simian-human immunodeficiency virus. J Virol. 1999;73(12):10199-10207.

60. Stiegler G, et al. A potent cross-clade neutralizing human monoclonal antibody against a novel epitope on gp41 of human immunodeficiency virus type 1 . AIDS Res Hum Retroviruses. 2001;17(18):1757-1765.

61. Purtscher M, et al. A broadly neutralizing human monoclonal antibody against gp41 of human immunodeficiency virus type 1 . AIDS Res Hum Retroviruses. 1994;10(12):1651-1658.

62. Purtscher M, et al. Restricted antigenic variability of the epitope recognized by the neutralizing gp41 antibody 2F5. AIDS. 1996;10(6):587-593.

63. Gorny MK, Gianakakos V, Sharpe S, Zolla-Pazner S. Generation of human monoclonal antibodies to human immunodeficiency virus. Proc Natl Acad Sci U S A. 1989;86(5):1624-1628.

64. Tyler DS, et al. Identification of sites within gp41 that serve as targets for antibody-dependent cellular cytotoxicity by using human monoclona antibodies. J Immunol. 1990;145(10):3276-3282.

65. Xu JY, Gorny MK, Palker T, Karwowska S, ZollaPazner S. Epitope mapping of two immunodominant domains of gp41, the transmembrane protein of human immunodeficiency virus type 1 , using ten human monoclonal antibodies. J Virol. 1991;65(9):4832-4838.

66. Robinson WE, Gorny MK, Xu JY, Mitchell WM, Zolla-Pazner S. Two immunodominant domains of gp41 bind antibodies which enhance human immunodeficiency virus type 1 infection in vitro. J Virol. 1991;65(8):4169-4176.

67. Kaslow RA, Ostrow DG, Detels R, Phair JP, Polk $\mathrm{BF}$, Rinaldo CR. The Multicenter AIDS Cohort Study: rationale, organization, and selected characteristics of the participants. Am J Epidemiol. 1987;126(2):310-318.

68. Detels R, et al. The Multicenter AIDS Cohort Study, 1983 to .... Public Health. 2012;126(3):196-198.

69. Takahashi K, et al. Plasmacytoid dendritic cells sense hepatitis $\mathrm{C}$ virus-infected cells, produce interferon, and inhibit infection. Proc Natl Acad Sci US A. 2010;107 (16):7431-7436.

70. Wasilewski LN, et al. A hepatitis $\mathrm{C}$ virus envelope polymorphism confers resistance to neutralization by polyclonal sera and broadly neutralizing monoclonal antibodies. J Virol. 2016;90(7):3773-3782. 